Submitted to ApJ, 11/09/06

\title{
Mapping the Cosmological Confidence Ball Surface
}

\author{
Brent Bryan and Jeff Schneider \\ Department of Machine Learning, Carnegie Mellon University, 5000 Forbes Avenue, \\ Pittsburgh, PA 15213 \\ $\{$ bryanba, schneide\}@cs.cmu.edu \\ Christopher J. Miller \\ Cerro Tololo Interamerican Observatory, Casilla 603, La Serena, Chile \\ cmiller@noao.edu \\ Robert C. Nichol \\ Institute of Cosmology and Gravitation, University of Portsmouth, Portsmouth, PO1 2EG, \\ $U K$ \\ bob.nichol@port.ac.uk \\ and \\ Christopher Genovese and Larry Wasserman \\ Department of Statistics, Carnegie Mellon University, 5000 Forbes Avenue, Pittsburgh, PA \\ 15213 \\ \{genovese, larry\}@stat.cmu.edu
}

\begin{abstract}
We present a new technique to compute simultaneously valid confidence intervals for a set of model parameters. We apply our method to the Wilkinson Microwave Anisotropy Probe's (WMAP) Cosmic Microwave Background (CMB) data, exploring a seven dimensional space $\left(\tau, \Omega_{\mathrm{DE}}, \Omega_{\mathrm{M}}, \omega_{\mathrm{DM}}, \omega_{\mathrm{B}}, f_{\nu}, n_{s}\right)$. We find two distinct regions-of-interest: the standard Concordance Model, and a region with large values of $\omega_{\mathrm{DM}}, \omega_{\mathrm{B}}$ and $H_{0}$. This second peak in parameter space can be rejected by applying a constraint (or a prior) on the allowable values of
\end{abstract}


the Hubble constant. Our new technique uses a non-parametric fit to the data, along with a frequentist approach and a smart search algorithm to map out a statistical confidence surface. The result is a confidence "ball": a set of parameter values that contains the true value with probability at least $1-\alpha$. Our algorithm performs a role similar to the often used Markov Chain Monte Carlo (MCMC), which samples from the posterior probability function in order to provide Bayesian credible intervals on the parameters. While the MCMC approach samples densely around a peak in the posterior, our new technique allows cosmologists to perform efficient analyses around any regions of interest: e.g., the peak itself, or, possibly more importantly, the $1-\alpha$ confidence surface.

Subject headings: cosmology: cosmic microwave background - cosmology: cosmological parameters — methods: statistical

\section{Introduction}

The Cosmic Microwave Background (CMB) angular temperature power spectrum is the most widely utilized data set for constraining the cosmological parameters (Tegmark et al. 2001; Christensen et al. 2001; Verde et al. 2003; Spergel et al. 2003; Tegmark et al. 2004). This power spectrum, which statistically measures the distribution of temperature fluctuations as a function of scale, is comprised of at least two peaks thought to have been formed by sound wave modes inherent in the primordial gas during recombination. The locations, heights, and height-ratios of the peaks and valleys in the power spectrum can provide direct information about fundamental parameters of the universe, such as the space-time geometry, the fraction of energy density contained in the baryonic matter, and the cosmological constant (Miller et al. 2001). However, it is more common for cosmologists to compare the observed CMB power spectrum to a suite of cosmological models (e.g. CMBFast (Seljak \& Zaldarriaga 1996) and CAMB (Lewis et al. 2000)). These models require as input some minimal number of cosmological parameters, $d$, - typically $d=6$ or $d=7$.

Most CMB power spectrum parameter estimations to date have been done via Bayesian

techniques (e.g., Knox et al. (2001); Gupta \& Heavens (2002); Spergel et al. (2003); Jimenez et al. (2004); Dunkley et al. (2005)). For these techniques, the $d$-dimensional likelihood function is parametrically estimated and prior probabilities are assumed for each parameter. Then, a posterior probability distribution can be computed, and credible intervals can be found. However, unless the form of the prior is conjugate on the likelihood (which is atypical), computing the posterior involves estimating an integral over the entire space spanned by the prior. There are two basic approaches to solving this problem in the literature. Tegmark et al. 
(2001) approximates this integral explicitly, using an adaptive grid, where grid cells are more densely located in areas presumed to be important. Secondly, and more popularly, many authors have used Markov Chain Monte Carlo (MCMC) (e.g. Gupta \& Heavens (2002); Lewis \& Bridle (2002); Jimenez et al. (2004); Sandvik et al. (2004); Dunkley et al. (2005); Chu \& Knox (2005); Hajian (2006)), which tend to be much more efficient than grid based techniques, but are notoriously difficult to tune and test for convergence (Wasserman 2004).

While Bayesian techniques are used in the majority of work on CMB parameter estimation, there have also been undertakings to estimate cosmological parameters using frequentist techniques, such as $\chi^{2}$ tests (Gorski et al. 1993; White \& Bunn 1995; Padmanabhan \& Sethi 2001; Griffiths et al. 2001; Abroe et al. 2002) and Bayes risk analyses (Schafer \& Stark 2003). We present a novel frequentist method based upon a non-parametric fit to the data to estimate the smooth underlying power spectrum, as well as an error "ellipse" following the technique used in Miller et al. (2001) and Genovese et al. (2004). This confidence ball has a radius which is a function of the probability with which the true power spectrum is contained within the ball and the observed error estimates. The ball radius is independent of both the models to be fit, as well as the parameter ranges to be queried. Thus, we can take a vector of parameters, run it through our favorite CMB power spectrum generating model, and determine whether or not the model (and hence the parameter vector) lies within our confidence ball, without fixing a priori the model to be used, or the parameter ranges to be searched. We are interested in finding the set of parameter vectors which lie within the $1-\alpha$ confidence ball, for some confidence level (or probability of being incorrect), $\alpha$.

This is a statistically different style of "confidence" than the credible intervals or the "degree of belief" one obtains using Bayesian techniques. In particular, the Bayesian method answers the question "assuming a given model and prior distribution over the parameter space, what is the smallest range of a particular parameter from which I believe the next sample will be drawn with probability $1-\alpha$ ?" In contrast, the frequentist approach constructs a procedure for deriving confidence intervals that when applied to a series of data sets, traps the true parameters for at least $100(1-\alpha) \%$ of the data sets. For parametric models with large sample sizes, Bayesian and frequentist approaches are known to result in similar inferences. However, for high dimensional and non-parametric problems - such as estimating cosmological parameters from the CMB power spectrum - Bayesian methods may not yield accurate inferences (Wasserman 2004). In such cases, the Bayesian $95 \%$ credible interval may not contain the true value $95 \%$ of the time in a frequency sense.

Additionally, mapping a region of high likelihood points in parameter space is fundamentally a search problem. As MCMC methods are designed to sample and/or integrate a distribution, they are not necessarily good search algorithms in practice. In particular, 
a MCMC method "represents" a high-likelihood region by heavily sampling that region an expensive proposition when using CMBFast. In contrast, a search algorithm that can directly observe the (normalized) likelihood of a sample will have no reason to spend more samples in the same location. In addition to describing a frequentist approach to computing confidence intervals for cosmological parameters, another significant contribution of this paper is the proposal of a new search algorithm for mapping confidence surfaces.

In this work, we utilize the non-parametric basis described by Miller et al. (2001) and Genovese et al. (2004) to constrain the set of cosmological models which fit the WMAP observations. At the same time, we must deal with the challenges posed in other frameworks namely: robustness of the algorithm, efficiency, and issues of convergence. A schematic outline of our technique is shown in Figure 1. In \$2, we briefly describe the data and cosmological models used, as well as the non-parametric technique (the bottom row of Figure 1). We then focus on a new algorithm to map the derived confidence ball into parameter space in $\$ 3.2$, sketched out on the top line of Figure 1. In \&4, we present results of our algorithm, and discuss challenges to accurately determine confidence intervals using any statistical approach. Finally, in \$5, we compare our method with commonly used inference techniques, and discuss the advantages of using the proposed approach.

\section{Methodology}

\section{1. $\quad$ Data \& Models}

We examine the CMB power-spectrum $\left(\hat{C}_{\ell}\right)$ as measured by the Wilkinson Microwave Anisotropy Probe's first-year data release (Bennett et al. 2003; Hinshaw et al. 2003; Verde et al. 2003)1, shown in Figure 2. Our approach is similar to that of other authors (e.g. Tegmark

\footnotetext{
${ }^{1}$ Available at http://lambda.gsfc.nasa.gov
}

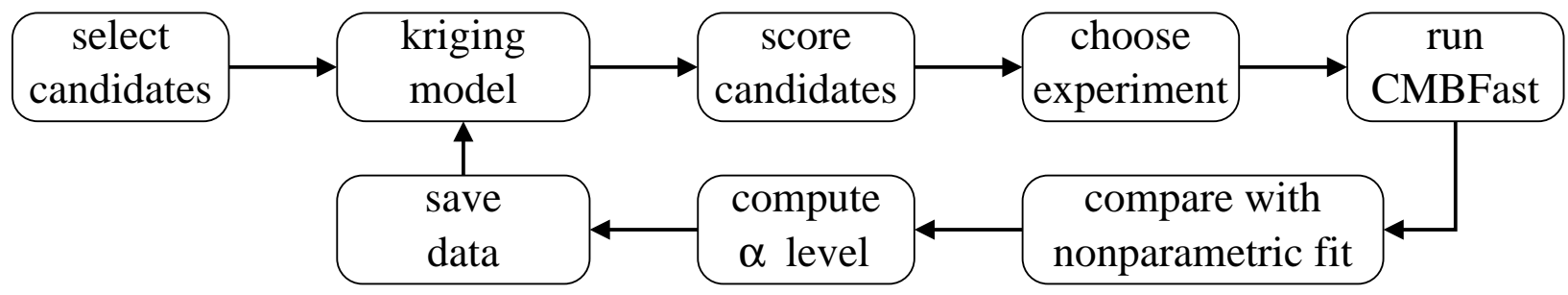

Fig. 1.- Schematic outline of our technique to constraint confidence intervals. 
(1999); Tegmark et al. (2001); Spergel et al. (2003)), who fit the observed CMB power spectrum to a suite of cosmological models. These models, while sophisticated and detailed, have numerous free parameters, some of which are difficult to ascertain (e.g. ionization depth, contribution of gravity waves). However, there are many codes available to compute CMB power spectrum, which trade off speed for accuracy and robustness.

Both CMBFast (Seljak \& Zaldarriaga 1996) and the related CAMB (Lewis et al. 2000) compute the CMB power spectrum by evolving the Boltzmann equation using a line of sight integration technique. While an order of magnitude faster than computing the full Boltzmann solution, this approach is still rather slow. One approach for reducing the computation time of CMBFast is to split the Boltzmann computation into low and high multipole moment portions, as the low and high multipoles are mostly independent (Tegmark et al. 2001). Using this method, ksplit, Tegmark et al. (2001) was able to reduce computation time by a factor of 10. Additionally, several approximate programs have been developed which are orders of magnitudes faster than CMBFast, including DASh (Kaplinghat et al. 2002), CMBWarp (Jimenez et al. 2004), and Pico (Fendt \& Wandelt 2006). In general, these programs gain great speedups by approximating the power spectrum with a regression function fit to predetermined sample points generated from simulators such as CMBFast. As a result, generating a hypothesis spectrum for a new set of parameters is a simple function evaluation, foregoing the computation of the Boltzmann equation entirely.

While using any one of these approximate methods or ksplit may seem appealing due to their computational efficiency, they do not have the desired accuracy and robustness (Seljak et al. 2003). These codes are only approximations. While fairly accurate around the concordance peak, their accuracy drops off drastically when computing models for parameter vectors slightly removed from the "accepted" cosmological models. Additionally, these codes are prone to failures when presented with parameter vectors that are not within a narrowly defined region around the concordance model (Fendt \& Wandelt 2006). According to the Pico website: "Since Pico's purpose is to be part of parameter estimation codes, we are mainly concerned with having the regression coefficients defined around the region of parameter space allowed by the data (mainly the WMAP3 data). Pico will not be able to compute accurate spectra and likelihoods away from this region, but it will warn you about this." Similarly, in many instances ksplit will hang on parameter vectors that are a short distance from the concordance peak. Since we are interested in finding the tightest possible confidence intervals for all regions of parameter space that can possibly fit the data, we do not want to be artificially restricted by our CMB simulator. Thus, we choose to compute the model CMB power spectra using CMBFast; while not the fastest code available CMBFast is accurate and reliable. 


\begin{tabular}{clr}
\hline Parameter & Description & Range \\
\hline \hline$\tau$ & optical depth & $0.0-1.2$ \\
$\Omega_{\mathrm{DE}}$ & dark energy mass fraction & $0.0-1.0$ \\
$\Omega_{\mathrm{M}}$ & total mass fraction & $0.1-1.0$ \\
$\omega_{\mathrm{DM}}$ & dark matter density & $0.01-1.2$ \\
$\omega_{\mathrm{B}}$ & baryon density & $0.001-0.25$ \\
$f_{\nu}$ & neutrino fraction & $0.0-1.0$ \\
$n_{s}$ & spectral index & $0.5-1.7$ \\
\hline
\end{tabular}

Table 1: Cosmological parameters and ranges searched.

Next, multipole covariance is estimated by using the covariance derived for the concordance model using code from Verde et al. (2003). We find that the computed variances match well with those found in the first-year data release, with only a slight (roughly 1.15) multiplicative offset. This constant factor offset was hinted at by the sub unity slope of the quantile-quantile plot of the variance weighted deviations between the data and the concordance model prediction, using the variances given in the WMAP data.

Spergel et al. (2006) show that the WMAP third year data are well described by a simple 6 parameter model: $\tau, H_{0}, \Omega_{\mathrm{M}}, \Omega_{\mathrm{B}}, \sigma_{8}, n_{s}$. In this paper, we use effectively the same model space as the simplified model in Spergel et al. (2006), except that we include the neutrino fraction and exclude $\sigma_{8}$. We made this change as we are not utilizing large-scale structure data, which is sensitive to $\sigma_{8}$. The resulting parameter vector $\mathbf{p}=\left(\tau, \Omega_{\mathrm{DE}}, \Omega_{\mathrm{M}}, \omega_{\mathrm{DM}}, \omega_{\mathrm{B}}, f_{\nu}, n_{s}\right)$ is similar to the model space searched by Tegmark et al. (2001). A description and considered range for each of these variables is presented in Table1; the parameter ranges considered here are slightly larger than those searched by Tegmark et al. (2001), due to our interest in mapping an observed secondary peak in parameter space. Note that $\Omega_{\mathrm{k}}=1-\Omega_{\mathrm{M}}-\Omega_{\mathrm{DE}}$. Moreover, the Hubble constant, $H_{0}$, is not an independent parameter, but given by

$$
\frac{H_{0}}{100}=h=\sqrt{\frac{\omega_{\mathrm{DM}}+\omega_{\mathrm{B}}}{\Omega_{\mathrm{M}}}}=\sqrt{\frac{\omega_{\mathrm{DM}}+\omega_{\mathrm{B}}}{1-\Omega_{\mathrm{k}}-\Omega_{\mathrm{DE}}}} .
$$

We denote the space spanned by $\mathbf{p}$ as $\mathcal{P}$. $\mathcal{P}$ is a seven dimensional hyper-rectangle where the range of the $j^{\text {th }}$ side corresponds to the range of the $j^{\text {th }}$ cosmological parameter of $\mathbf{p}$.

\subsection{Nonparametric Analysis}

We now provide a brief sketch of nonparametric data analysis, as it pertains to the CMB power spectrum. We follow the derivations given in Miller et al. (2001) and Genovese et al. 
(2004), and refer interested readers to those works. Our technique is designed to:

1. Compute a fit to the actual data which minimizes the sum of the bias and the variance between the fit and the data, taking into account the full covariance discussed in $\$ 2.1$. Errors are assumed to be Gaussian. This fit is effectively a smoothed version of the data.

2. Determine a confidence ellipse ball around the best fit for a given test level, $\alpha$.

3. Find all such vectors $s \in \mathcal{P}$ such that the power spectrum output by CMBFast for $s$ results in a model which is contained within the $1-\alpha$ confidence ball found in step 2 .

We now detail items 1 and 2, leaving the discussion of item 3 to $\S 3$,

\subsubsection{The Non-Parametric Fit}

Let $\ell \in\left[L_{\min }, \ldots, L_{\max }\right]$ denote a generic index of the CMB temperature power spectrum multipole, and $n=L_{\max }-L_{\min }+1$ be the total number of observed multipoles. We take $Y_{\ell}=\hat{C}_{\ell}$ to be the observations of the CMB where $x_{\ell}=\left(\ell-L_{\min }\right) /\left(L_{\max }-L_{\min }\right)$ and let $f\left(x_{\ell}\right) \equiv C_{\ell}$ denote the true power spectrum at multipole index $\ell$. We then solve the nonparametric regression problem:

$$
Y_{\ell}=f\left(x_{\ell}\right)+\epsilon_{\ell}, \quad \ell=L_{\min }, \ldots, L_{\max },
$$

where $\epsilon=\left(\epsilon_{L_{\min }}, \ldots, \epsilon_{L_{\max }}\right)$ are assumed Gaussian with known covariance matrix $\Sigma$ as described earlier. Henceforth, we will use $i=\ell-L_{\min }+1$ as an index. Nonparametric analysis is based on the notion of estimating a function without forcing it to fit some finite-dimensional parameter form (e.g. a Normal distribution), by smoothing the data in such a way to balance the bias and variance. In this work, we use orthogonal series regression to estimate $f$, expanding $f$ as a cosine basis:

$$
f(x)=\sum_{j=0}^{\infty} \mu_{j} \phi_{j}(x)
$$

where

$$
\phi_{j}(x)= \begin{cases}1 & \text { for } j=0 \\ \sqrt{2} \cos (\pi j x) & \text { for } j=1,2,3, \ldots\end{cases}
$$

and the $\mu_{j}$ 's are the coefficients for each basis component. If $f$ is smooth, then $\mu_{j}$ will decay rapidly as $j$ increases. That is, if $f$ is smooth, then there are little or no high frequency fluctuations in $f$ and hence $\mu_{j} \simeq 0$. Thus, $\sum_{j=n+1}^{\infty} \mu_{j}^{2}$ will be negligible, and we can approximate 
the infinite sum as $f(x) \approx \sum_{j=0}^{n} \mu_{j} \phi_{j}(x)$. Let

$$
Z_{j}=\frac{1}{n} \sum_{i=1}^{n} Y_{i} \phi_{j}\left(X_{i}\right)
$$

for $j=0,1, \ldots n$. Then $Z$ is approximately normal distributed with mean $\mu$ and covariance $B / \sqrt{n}=U \Sigma U^{T} / \sqrt{n}$, where $U$ is the cosine basis transformation matrix.

In order to obtain an even smoother estimate of $f$, we damp out the higher frequencies using shrinkage estimators. We let $\hat{\mu}_{j}=\lambda_{j} Z_{j}$ where $1 \geq \lambda_{0} \geq \lambda_{1} \geq \cdots \geq \lambda_{n} \geq 0$ are shrinkage coefficients. The estimate of $f$ is now

$$
\hat{f}(x)=\sum_{j=0}^{n} \hat{\mu}_{j} \phi_{j}(x)=\sum_{j=0}^{n} \lambda_{j} Z_{j} \phi_{j}(x) .
$$

Following Genovese et al. (2004), we use a special case of monotone shrinkage in which

$$
\lambda_{j}= \begin{cases}1 & \text { for } j \leq J \\ 0 & \text { for } j>J\end{cases}
$$

for some integer $J \in[0, n]$. We will show how to find $J$ shortly. Using the monotone shrinkage scheme described above, the estimate of $f$ becomes

$$
\hat{f}(x)=\sum_{j=0}^{J} Z_{j} \phi_{j}(x) .
$$

The squared error loss as a function of $\hat{\lambda}=\left(\hat{\lambda}_{0}, \hat{\lambda}_{1}, \ldots, \hat{\lambda}_{n}\right)$ is

$$
L_{n}(\hat{\lambda})=\int_{0}^{1}\left(\frac{\hat{f}(x)-f(x)}{\sigma(x)}\right)^{2} d x \approx \sum_{j=1}^{n}\left(\frac{\mu_{j}-\hat{\mu}_{j}}{\sigma_{j}}\right)^{2}
$$

where $\sigma^{2}(x)$ is the variance of $f$, and $\sigma_{j}^{2}$ are the observed variances of the power spectrum (the elements on the diagonal of $\Sigma$ ). Meanwhile, the risk is given by

$$
R(\lambda)=\mathrm{E}\left[\int_{0}^{1}\left(\frac{\hat{f}(x)-f(x)}{\sigma(x)}\right)^{2} d x\right] \approx \frac{J}{n}+\sum_{j=J}^{n} \frac{\mu_{j}^{2}}{\sigma_{j}^{2}}
$$

We choose $J$ to minimize the Stein's unbiased risk estimate

$$
\hat{R}=Z^{T} \bar{D} W \bar{D} Z+\operatorname{trace}(D W D B)-\operatorname{trace}(\bar{D} W \bar{D} B)
$$


where $D$ and $\bar{D}=1-D$ are diagonal matrices with 1's in the first $J$ and last $n-J$ entries respectively, $B$ is the covariance of $Z$, and $W_{j k}=\sum_{\ell} \Delta_{j k \ell} / \sigma_{\ell}$ and

$$
\begin{aligned}
\Delta_{j k \ell} & =\int_{0}^{1} \phi_{j} \phi_{k} \phi_{\ell} \\
& =\left\{\begin{array}{cl}
1 & \text { if } \#\{j, k, l=0\}=3 \\
0 & \text { if } \#\{j, k, l=0\}=2 \\
\delta_{j k} \delta_{0 \ell}+\delta_{j \ell} \delta_{0 k}+\delta_{k \ell} \delta_{0 j} & \text { if } \#\{j, k, l=0\}=1 \\
\frac{1}{\sqrt{2}}\left(\delta_{\ell, j+k}+\delta_{\ell,|j-k|}\right) & \text { if } \#\{j, k, l=0\}=0
\end{array} .\right.
\end{aligned}
$$

Beran \& Dümbgen (1998) showed that $\hat{R}(\lambda)$ is asymptotically, uniformly close to $R(\lambda)$ when using monotone shrinkage coefficients and $\sigma(x)=1$. Genovese et al. (2004) extended this result to the heteroskedastic case used here.

In Figure 2, we compare our non-parametric fit to the WMAP data to a model-based fit from Spergel et al. (2003). Points in the figure depict the first year WMAP data. Error bars are omitted for clarity. The full estimated covariance, $\Sigma$, is used in both the Spergel et al. (2003) model fit and the Genovese et al. (2004) non-parametric fit.

\subsubsection{The Confidence Ball}

After we perform the non-parametric fit, we need to quantify the uncertainty to make statistical inferences. We use the Beran-Dümbgen pivot method (Beran \& Dümbgen 1998; Beran 2000) to derive valid confidence intervals. This method relies on the weak convergence of the "pivot process" - $B_{n}(\hat{\lambda})=\sqrt{n}\left(L_{n}(\hat{\lambda})-\hat{R}(\hat{\lambda})\right)$ - to a Normal $\left(0, \tau^{2}\right)$ distribution for some $\tau^{2}>0$; a derivation of $\hat{\tau}_{n}$ can be found in Appendix $\mathrm{A}$, taken from Appendix 3 of Genovese et al. (2004). Using the convergence of the pivot process, we can compute a confidence ellipse for the basis coefficients with a "radius" given by:

$$
\mathcal{D}_{n}=\left\{\mu: \sum_{i=1}^{n}\left(\frac{\hat{\mu}_{i}-\mu_{i}}{\sigma_{i}}\right)^{2} \leq \frac{\hat{\tau}_{n} z_{\alpha}}{\sqrt{n}}+\hat{R}\left(\hat{\lambda}_{n}\right)\right\}
$$

where the best fit to the data is represented by $\hat{\mu}_{i}$, the function being tested (whether it is within some confidence ball) is $\mu_{i}$, and the level of the confidence ball is determined by $z_{\alpha}$, the upper $\alpha$ quantile of a standard Normal distribution.

Therefore, using the central limit theorem, we have

$$
\mathcal{B}_{n}=\left\{f(x)=\sum_{j=0}^{n} \mu_{j} \phi_{j}(x): \mu \in \mathcal{D}_{n}\right\}
$$




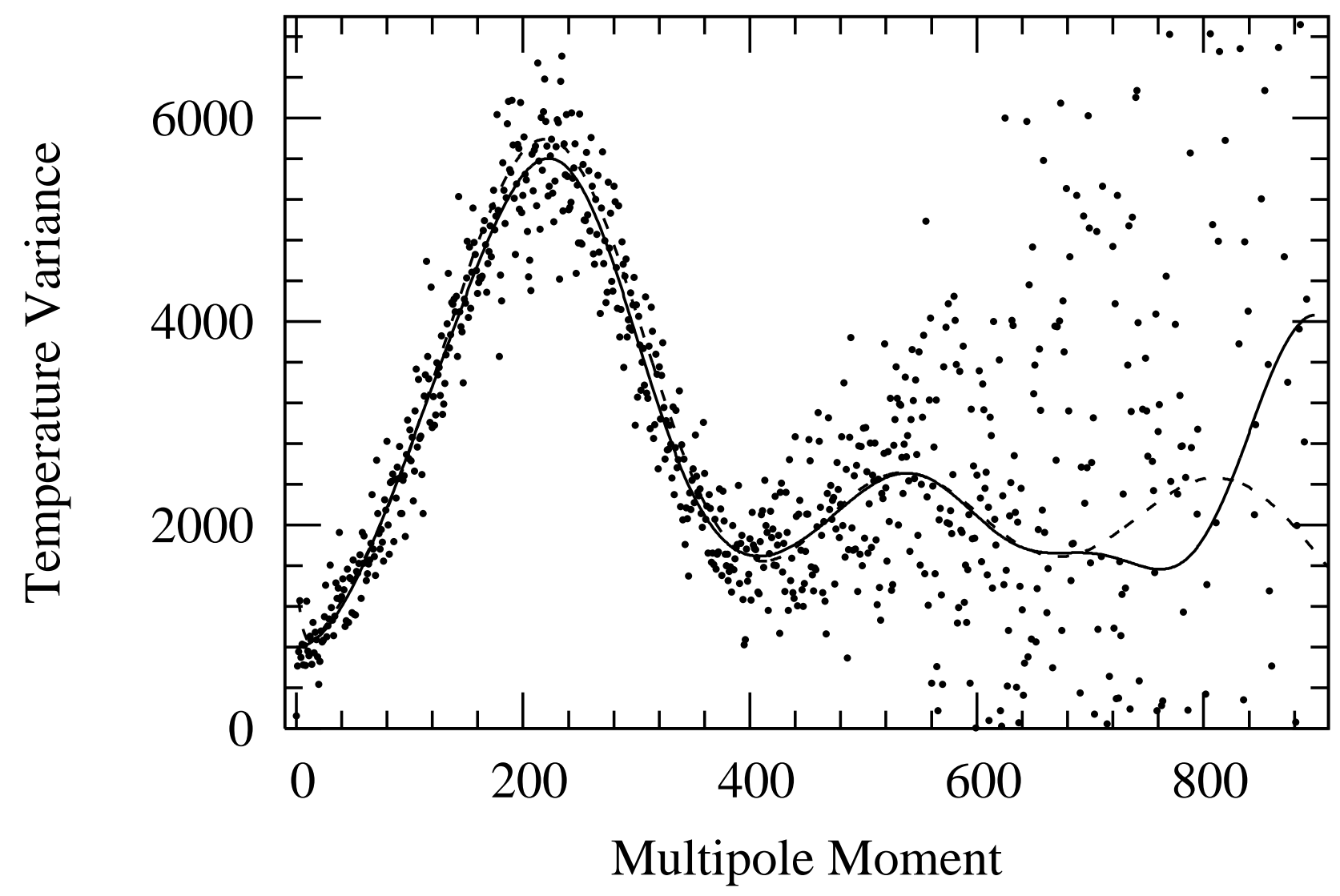

Fig. 2.- Comparison of our nonparametric fit of the CMB power-spectrum (solid) with Spergel et al. (2003) parametric fit (dashed). First-year WMAP data (dots) are shown without errors for clarity. 
is an asymptotic $1-\alpha$ confidence set for $f$.

Thus, to determine if any given vector $s \in \mathcal{P}$ is within our confidence ball, we merely have to run our cosmological model to compute the resulting power spectrum, $\hat{f}(s)$, and

check to see if $\hat{f}(s) \in \mathcal{B}_{n}$. This can be easily done by using Equation 3 to check whether the sum of squares of $\hat{\mu}$ and $\mu$ are less than a constant given on the right-hand side of Equation 3. As shown in Figure 11, as the radius increases, so does the size of the confidence set (and $\alpha$ decreases). Thus, a 95\% (or $\alpha=0.05$ ) confidence region has a larger "radius" than does a $67 \%$ (or $\alpha=0.33$ ) confidence region. Moreover, a $1-\alpha$ confidence ball strictly contains all confidence balls with smaller values of $1-\alpha$.

Since the dimensionality of our space is large, it is difficult to visualize the confidence region that surrounds the non-parametric fit. However, we can show examples of functions which live inside (or outside) our confidence region by calculating their distance from the nonparametric fit to the data. In Figure 3, we show a "ribbon" plot for $\omega_{\mathrm{B}}$ around the concordance model. This figure is generated by setting all of the cosmological parameters to their concordance values and then slowly evolving $\omega_{\mathrm{B}}$ from 0.012250 to 0.036750 to depict the range of temperature spectra allowed due to uncertainty of $\omega_{\mathrm{B}}$. The black curves are cosmological models which live within the $95 \%$ confidence ball, while gray curves are models that do not. As can be seen in this figure, the shape of the confidence region is not simply a band of constant width surrounding the best fit. It is, in fact, a very complicated, possibly disconnected surface in our high-dimensional parameter space. It is this confidence surface that we wish to map in detail.

\section{Mapping the Confidence Surfaces}

While theoretically Equation 4 exactly gives us the $1-\alpha$ confidence bound for any functional of the data, it is not trivial to compute what these bounds are. While it is easy to use Equation 4 to compute whether or not a given model is within the confidence ball, the method outlined in $\$ 2.2$ does not provide a way to easily compute all those spectrum that lie within that ball.

Concretely, when we test if a CMB power spectrum lies within the ball, we compare the given spectrum with the non-parametric fit found above, by computing a variance weighted sum of squares between the given spectrum and the regressed model. We call this weighted sum of squares the test spectrum's "distance". If we are given a model which results in a test spectrum whose distance is greater than the radius of our confidence ball, then we can reject the test spectrum (and its associated parameter vector) at the $1-\alpha$ level. If not, then 


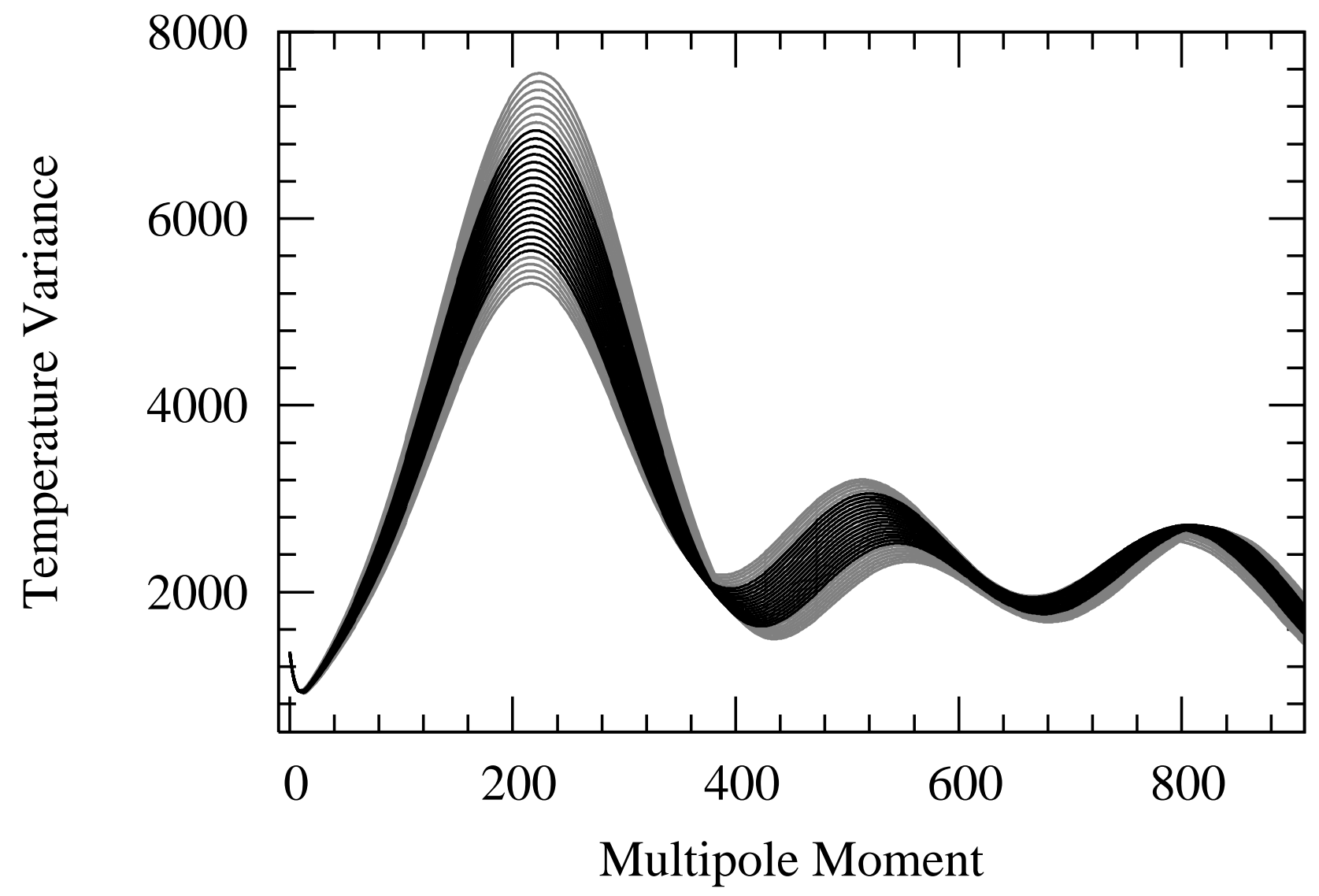

Fig. 3.- A "ribbon" plot depicting the effect of varying $\omega_{\mathrm{B}}$ while all other parameters remain fixed (at concordance values). Black lines indicate those models which are contained within a $95 \%$ confidence ball, while gray lies indicate those models rejected by the hypothesis that the model and the regressed fit are the same. 
our test does not have the power to distinguish between the regressed model and our test model. Note that we are taking a $\sim 900$ element spectrum and compressing it to a scalar. Thus, there are many models - possibly representing vastly different spectra — that may result in exactly the same distance value. For the hypothesis test that the fitted function and regressed models are derived form the same distribution, we will draw the same conclusion for all models with the same distance values. Either all models with a particular distance score can be rejected or none can. For a given confidence ball radius, we could compute (possibly with some discrete approximation) all of the possible CMB power spectra that have distances equal to the confidence radius. However, we are unaware of an easy way to determine the cosmological parameters of a power spectrum given only the power spectrum itself. That is, we do not have a method to easily invert CMBFast.

Of course, one solution would be to grid the parameter space, and run a model for each grid cell. We could then use these models to approximate the mapping between parameter vectors and confidence level using, for instance, a simple linear approximator. As noted in §1, such an approach is far too slow, explaining why Tegmark et al. (2001) use both adaptive grids and a modified version of CMBFast. Instead, we suggest an adaptive approach, which allows us to determine confidence intervals of our cosmological parameters more quickly and accurately. In particular, we are able to quickly refine our approximating surface in the areas of interest - those near the confidence ball's radius - while ignoring the uninteresting regions. This allows us to obtain estimates of the $1-\alpha$ confidence intervals of our cosmological parameters much more efficiently.

\subsection{Modeling Known Experiments}

The combination of CMBFast and the confidence ball method gives us a scoring function $f: \mathcal{P} \rightarrow \mathbb{R}$, which takes an input vector of parameters $(s \in \mathcal{P})$ and returns a distance value. This is accomplished by plugging the cosmological parameter values of $s$ into CMBFast to compute a model power spectrum, and then comparing this model spectrum with our nonparametric fit to the observed power spectrum using Equations 3 and 4 , Given a particular $1-\alpha$ confidence ball radius, $t$, we want to find the set of points, $\mathcal{S}(\mathcal{S} \subseteq \mathcal{P})$, that have distances to the regressed fit of the data less than or equal to the confidence ball radius: $\{s \in \mathcal{S} \mid s \in \mathcal{P}, f(s) \leq t\}$. Since we can not easily invert $f$ - that is to say CMBFast - we must deduce $\mathcal{S}$ by carefully sampling the points in $\mathcal{P}$.

For CMBFast, the cost to compute $f(s)$ given $s$ can be significant: computing power spectra away from the concordance model can take 5 to 15 minutes. Thus, care should be taken when choosing the next experiment, as picking optimum points can reduce the run time 
of the algorithm by orders of magnitude. Thus, it is preferable to analyze current knowledge about the underlying function and select experiments which quickly refine the estimate of the distance function around the confidence ball radius. There are several methods one could use to create a model of the data, notably some form of parametric regression. However, we chose to approximate $f(s)$ using Gaussian process regression, as other forms of regression may smooth the data, ignoring subtle features of the function that may become pronounced with more data. A Gaussian process is a non-parametric form of regression. Predictions for unobserved points are computed by using a weighted combination of the function values for those points which have already been observed, where a distance-based kernel function is used to determine the relative weights. These distance-based kernels generally weight nearby points significantly more than distance points. Thus, assuming the underlying function is continuous, Gaussian processes will perfectly describe the function given an infinite set of unique data points.

In this work, we use ordinary kriging, a form of Gaussian processes that assumes that the semi-variance, $\mathcal{K}(\cdot, \cdot)$, between two points is a linear function of their distance (Cressie 1991); for any two points $s_{i}, s_{j} \in \mathcal{P}$,

$$
\mathcal{K}\left(s_{i}, s_{j}\right)=\frac{k}{2} \mathrm{E}\left[\left(f\left(s_{i}\right)-f\left(s_{j}\right)\right)^{2}\right]
$$

where $k$ is a constant - known as the kriging parameter — which is an estimate of the maximum magnitude of the first derivative of the function. Therefore, the expected semivariance between two points, $s_{i}, s_{j} \in \mathcal{P}$ is given by

$$
\begin{aligned}
\gamma\left(s_{i}, s_{j}\right) & =E\left(\mathcal{K}\left(s_{i}, s_{j}\right)\right)=k \mathcal{D}\left(s_{i}, s_{j}\right)+c \\
& =k\left[\sum_{\ell=1}^{d} \alpha_{\ell}^{2}\left(s_{i \ell}-s_{j \ell}\right)^{2}\right]^{1 / 2}+c
\end{aligned}
$$

where $\mathcal{D}(\cdot, \cdot)$ is a distance function defined on the parameter space $\mathcal{P}$ and $c$ is the observed variance (e.g. experimental noise) when repeatedly sampling the function $f$ at the same location. We have found that using a simple weighted distance function where each dimension is linearly scaled by the parameter $\alpha_{\ell}$, as depicted in the previous equation, reasonably ensures that parameters are given equal consideration given their disparate values and derivatives. For our analysis, we adjusted the $\alpha_{\ell}$ 's to ensure that the maximum derivative along each dimension was approximately 1 during the sampling process. Additionally, while the simulations computed by CMBFast are deterministic, we shall see in $\$ 4.2$ that there is some inherent noise in the computations; thus we conservatively set $c=1 \times 10^{-5}$ in our analysis.

For the Gaussian process framework, sampled data are assumed to be Normally distributed with means equal to the true function and variance given by the sampling noise. 
Moreover, a combination of any subset of these points results in a Normal distribution. Thus, we can use the observed set of data, $\mathcal{A} \subset \mathcal{P}$, to predict the value of $f$ for any $s_{q} \in \mathcal{P}$. This query point, $s_{q}$, will be Normally distributed, $\left(N\left(\mu_{s_{q}}, \sigma_{s_{q}}\right)\right)$, with mean and variance given by

$$
\begin{aligned}
\mu_{s_{q}} & =\bar{f}_{\mathcal{A}}+\Sigma_{\mathcal{A} q}^{T} \Sigma_{\mathcal{A} \mathcal{A}}^{-1}\left(f_{\mathcal{A}}-\bar{f}_{\mathcal{A}}\right) \\
\sigma_{s_{q}}^{2} & =\Sigma_{\mathcal{A} q}^{T} \Sigma_{\mathcal{A} \mathcal{A}}^{-1} \Sigma_{\mathcal{A} q}
\end{aligned}
$$

where the elements of the matrix $\Sigma_{\mathcal{A} \mathcal{A}}$ and arrays $\Sigma_{\mathcal{A} q}$ and $f_{\mathcal{A}}-\bar{f}_{\mathcal{A}}$ are given by

$$
\begin{aligned}
\Sigma_{\mathcal{A A}}[i, j] & =\gamma\left(a_{i}, a_{j}\right) \\
\Sigma_{\mathcal{A} q}[i] & =\gamma\left(a_{i}, s_{q}\right) \\
\left(f_{\mathcal{A}}-\bar{f}_{\mathcal{A}}\right)[i] & =f\left(s_{i}\right)-\bar{f}_{\mathcal{A}} \\
\bar{f}_{\mathcal{A}} & =\frac{1}{|\mathcal{A}|} \sum_{i=1}^{|\mathcal{A}|} f\left(a_{i}\right)
\end{aligned}
$$

and the $a_{i}$ 's and $a_{j}$ 's are the observed data used to make an inference: $a_{i}, a_{j} \in \mathcal{A}, 0 \leq i, j \leq$ $|\mathcal{A}|$.

As given, for a set of $n$ observed points $(|\mathcal{A}|=n)$, prediction with a Gaussian process requires $O\left(n^{3}\right)$ time, as an $n \times n$ linear system of equations must be solved. However, for many Gaussian process - and ordinary kriging in particular - the correlation between two points decreases as a function of distance. Thus, the full Gaussian process model can be approximated well by a local Gaussian process, where only the $k$ nearest neighbors of the query point are used to compute the prediction value; this reduces the computation time to $O\left(k^{3}+k \log (n)\right)$ per prediction, since $O(k \log (n))$ time is required to find the k-nearest neighbors using spatial indexing structures such as balanced kd-trees.

\subsection{Algorithm}

There are many well-known heuristics for computing where best to perform the next experiment using a regression model, such as that derived in 3.1. Sampling strategies include

picking the point with the largest variance (MacKay 1992; Guestrin et al. 2005), entropy or information gain.

Sampling points based solely on variance is common in active learning methods whose goal is to map out an entire function, as this will minimize the expected error for prediction. Moreover, the model variance predicted by local ordinary kriging is linear in the distance to the nearest neighbors. As such, this strategy chooses points that are far from areas currently 
searched, and thus will not get stuck in a specific location in parameter space. However, this strategy is known to over sample boundary regions (MacKay 1992), and ultimately samples the space evenly like a grid. It is likely that large regions of the input space, $\mathcal{P}$, fall well outside the confidence ball radius. In the progression of the algorithm, points in these regions may have large variances but still not be within 2 or more standard deviations of the boundary; these points are very unlikely to be near the confidence ball radius. Hence, a strategy that samples the entire space evenly, using either a grid or a variance metric, can be extremely inefficient for mapping function boundaries.

Information gain heuristics are also popular in the machine learning community. However in a continuous parameter space, computing the effect of adding a new point is prohibitively expensive. Specifically, calculating the information gain of a proposed sample requires integrating the difference between the current model and expected result of the proposed sample over all space. Since our function approximator has only local support for predictions, we can reduce this integral down to the local region. However on this local region, computing the expected value of the model requires multiple matrix inversions to account for differences in the 100 nearest neighbors over the local region. Even approximating this integral with a (small) finite sum, was found to be prohibitively expensive. Instead, we use a strategy that is a combination of entropy and variance (both easy to compute), and is related to information gain. For more discussion on sampling strategies and their performance, we refer interested readers to Bryan et al. (2005).

The method we use here, named "Straddle", combines the desire to search the entire input space with that of refining our estimate around known interesting regions. We do this by picking points that the model predicts are both close to the boundary and have large variances using the following heuristic:

$$
\operatorname{straddle}\left(s_{q}\right)=1.96 \sigma_{s_{q}}-\left|\mu_{s_{q}}-t\right| .
$$

Note that the straddle heuristic chooses those points with large variances which straddle the boundary. In particular, if a point is near the boundary, then $\mu_{s_{q}} \simeq t$ and this metric is equivalent to a variance-only metric, choosing points that are distant from one another. However, if the point is not on the boundary, then its score drops off proportionally to the distance from the boundary. The straddle score for a point may be negative, which indicates that we predict that the probability that the point is on a boundary is less that five percent. Note that the straddle algorithm scores points highest that are both unknown and near the boundary, and thus gives scores that intuitively are similar to that of information gain.

Our sampling strategy then consists of four steps. First we model our current knowledge using the Gaussian process described in $\$ 3.1$. We then choose a set of candidate points randomly from the input space and compute their mean and variances using the Gaussian 
process model. Next, we score these points using the Straddle heuristic, and select the highest scoring point. Finally, we run the chosen point through CMBFast and add use the result to refine our Gaussian process model.

Ideally, we would like to analyze the entire input space, and pick experiments in such a manner that minimizes the number of experiments necessary. However, as our input space is infinite (the parameters are continuous), we need a heuristic to quickly generate a large, but not unwieldy set of candidate points. A priori, we have no information about the function we are trying to model. Therefore, in order to ensure that all boundary segments of the true function are found (assuming sufficient experimentation), it is necessary that candidate points be chosen such that all infinitesimal hyper-rectangles in the input space have non-zero probabilities of being chosen. We therefore choose candidate points uniformly at randomly from the input space, as this satisfies the probability constraint and is extremely quick. We note that bad candidate points will be discarded when their straddle scores are computed, and pose no problem for the algorithm.

\section{Results}

Using the algorithm described in $₫ 3.2$, we have sampled just over 1.2 million CMBFast models creating a "primary" data set. Additionally, we sampled another 100 thousand models uniformly at random throughout the parameter space. From the randomly sampled data, we find that less than $0.1 \%$ of the parameter space searched is within the $2 \sigma$ confidence ball; that is, our set of acceptable models (those within $2 \sigma$ ) exclude $99.97 \%$ of all possible models defined in Table 1. However, the method we use to generate parameter vectors results in only $54 \%$ of the points being rejected by the hypothesis that the model and the regressed fit are the same. Thus, by actively searching through the space, we are able to identify and efficiently map regions of interest, while ignoring large areas of parameter space that result in models below the $2 \sigma$ level. In $\$ 5.2$ we will see that our method is much more data efficient than typical Bayesian methods.

\subsection{Confidence Interval Projections}

The result of running the 1.2 million models contained in the primary data set is a set of disjoint, seven dimensional "confidence regions" in parameter space which contain all models that fall within our $1-\alpha$ confidence ball. In each of these regions, the confidence interval for a particular parameter is given by the range of values that parameter takes in that region. 

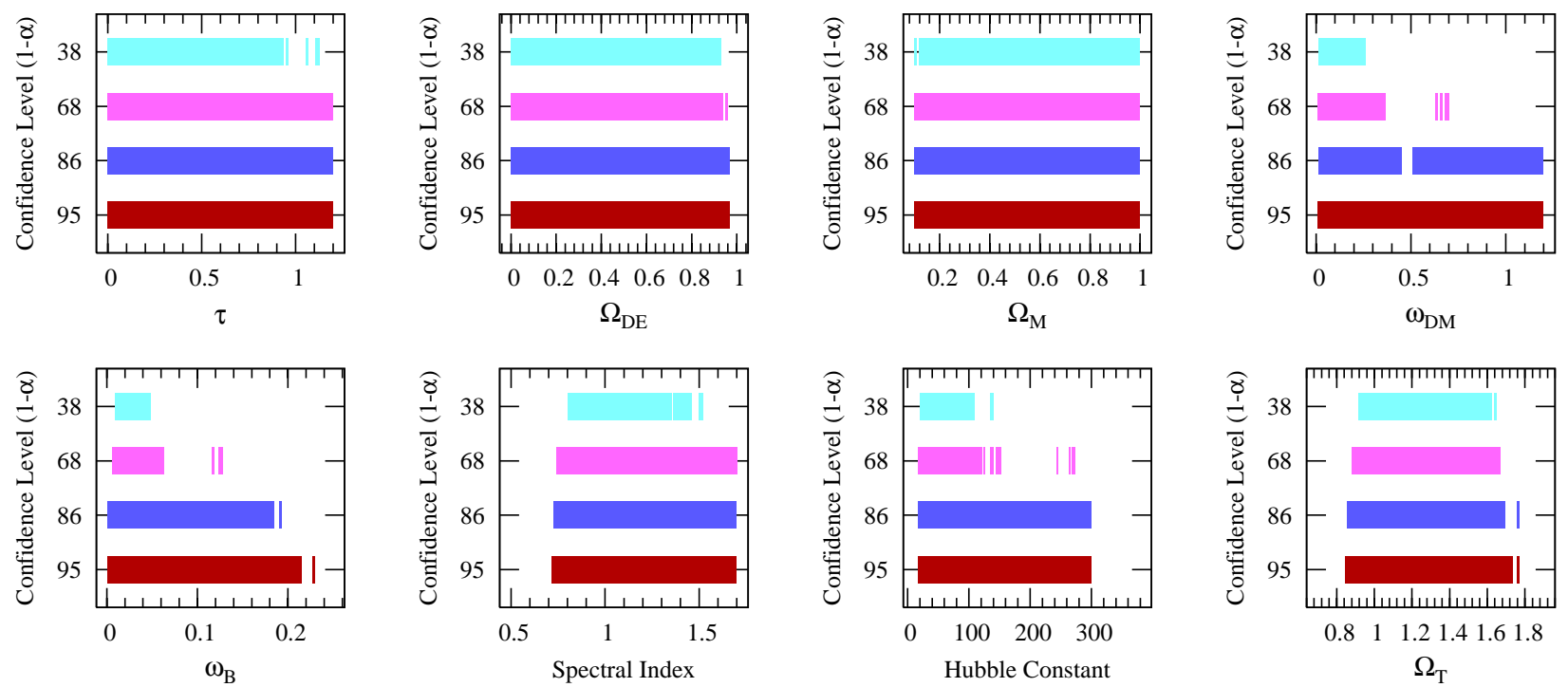

Fig. 4.- Jointly valid confidence intervals for our cosmological parameters for four values of $1-\alpha$, corresponding to $\frac{1}{2} \sigma, \sigma, 1 \frac{1}{2} \sigma$ and $2 \sigma$ confidence levels, respectively. Areas of solid color indicate values for the given parameter that contain the true value of cosmological parameter with probability $1-\alpha$, regardless of the values of the remaining 6 parameters. See the electronic edition of the Journal for a color version of this figure.

Thus, the confidence interval for a particular parameter will be a function of which sets of regions we consider.

If we put no restrictions on the values of the other 6 parameters, then the confidence interval of a parameter will be the union of the confidence intervals for that parameter for all confidence regions. We plot these unrestricted confidence intervals in Figure 4 for four values of $1-\alpha$. Intuitively, Figure 4 can be interpreted as stating that for any value of a parameter that lies within the depicted $1-\alpha$ confidence interval, there exists at least one combination of the remaining six parameters such that the resulting parameter vector lies within one of the $1-\alpha$ confidence regions.

In Figure 5 we depict results of interactions between pairs of parameters on the computed confidence regions. As with the 1D projections in Figure 4, points in Figure 5 which are denoted to be within the $1-\alpha$ confidence ball, are points where given the particular values of the two fixed cosmological parameters - those being explicitly plotted on the $x$ and $y$ axes, - there exists some values for the other 5 parameters such that the resulting parameter vector is within the $1-\alpha$ confidence region. While some plots show that most combinations of the fixed parameters are within the $95 \%$ confidence ball providing minimal constraints on 

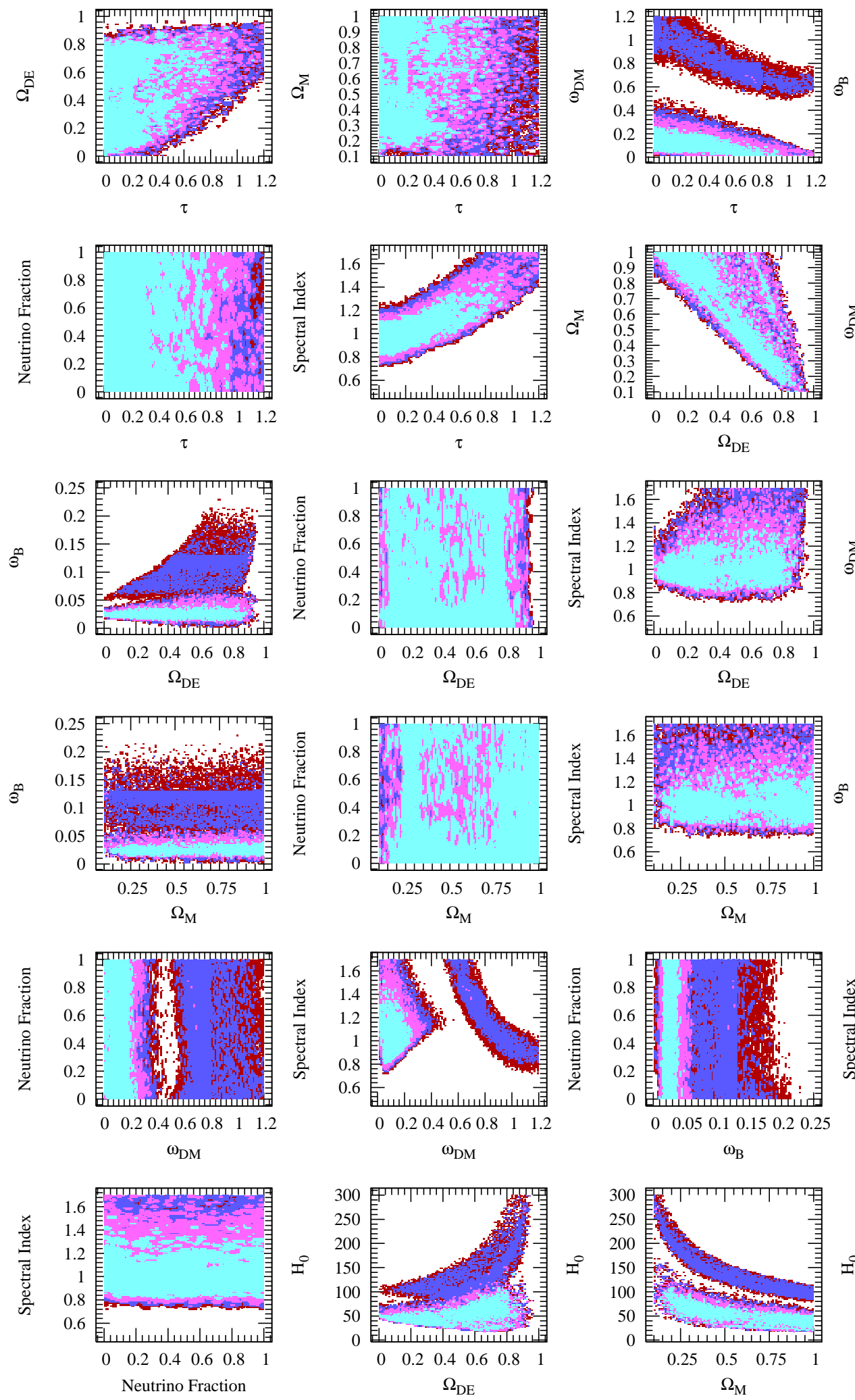
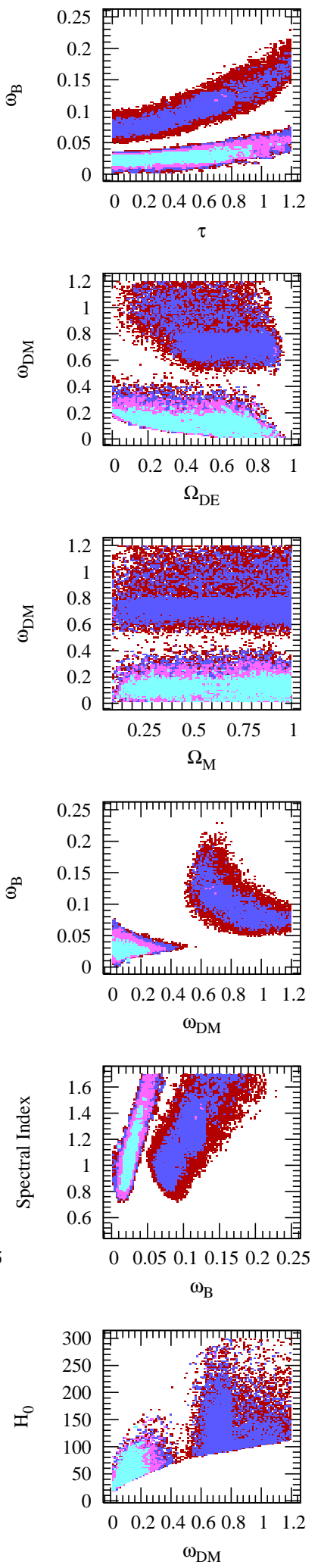

Fig. 5.- Jointly valid confidence regions for pairs of cosmological parameters, where the colors cyan, magenta, blue and red correspond to $\frac{1}{2} \sigma, \sigma, 1 \frac{1}{2} \sigma$ and $2 \sigma$, confidence levels respectively. Areas of solid color indicate values for the given pair of fixed (plotted) parameters that contain the true value of cosmological parameter with probability $1-\alpha$, regardless of 

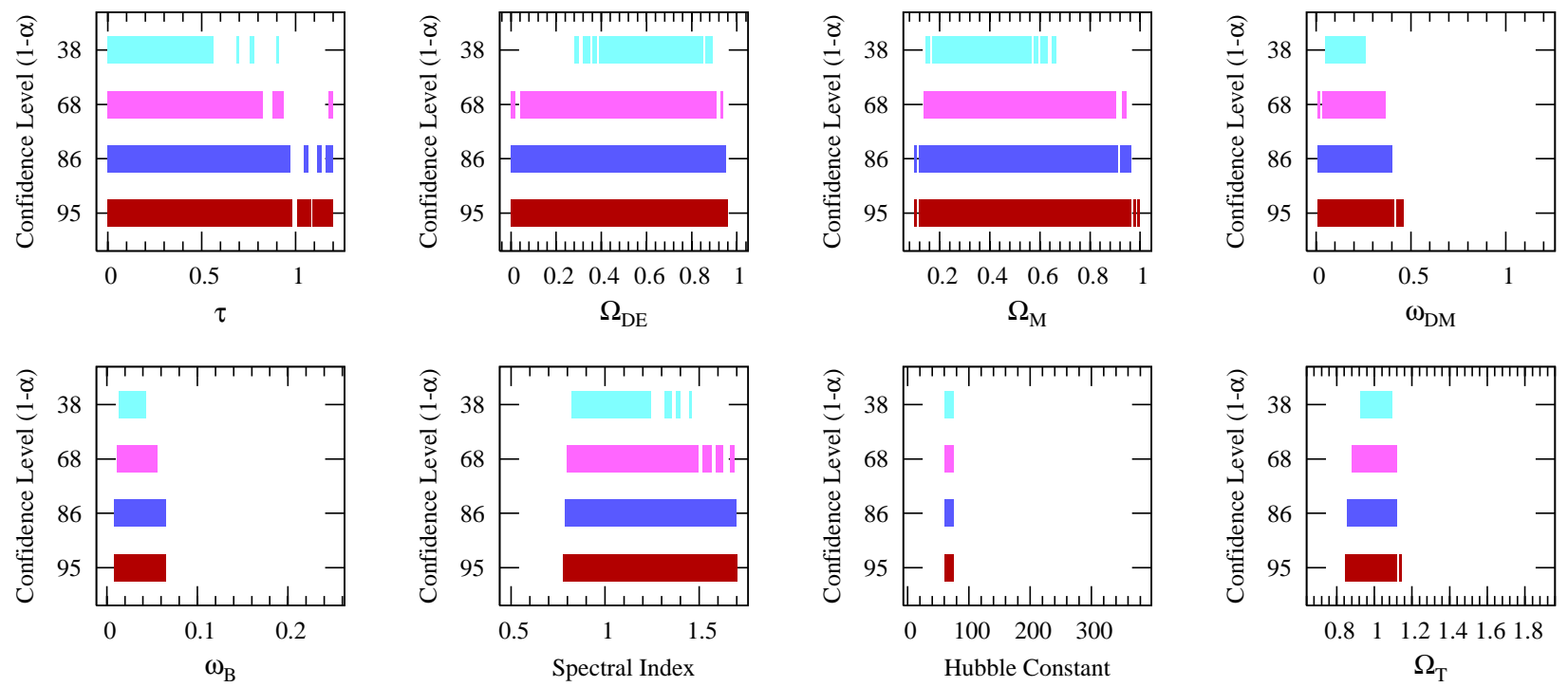

Fig. 6. - Jointly valid confidence intervals for our cosmological parameters, where we assume that that the value of $H_{0}$ is between 60 and $75 \frac{\mathrm{km} / \mathrm{s}}{\mathrm{Mpc}}$. Areas of solid color indicate values for the given parameter that contain the true value of cosmological parameter with probability $1-\alpha$, regardless of the values of the remaining 6 parameters. See the electronic edition of the Journal for a color version of this figure.

parameters describing the Universe, others, such as $\omega_{\mathrm{DM}}$ versus $\omega_{\mathrm{B}}\left(4^{\text {th }}\right.$ row, $4^{\text {th }}$ column$)$, show strong constraints.

Areas in Figure 5 which are blank (white), are areas that are rejected at the $95 \%$ confidence level; for these combinations of fixed parameters, there exists no combination of the other five parameters, such that the resulting vector is within any of our confidence regions. In particular, the plot of $\Omega_{\mathrm{DE}}$ versus $\Omega_{\mathrm{M}}\left(2^{\text {nd }}\right.$ row, $3^{\text {rd }}$ column) illustrates that $\Omega_{\text {Total }} \gtrsim 0.9$, while the plot of $\omega_{\mathrm{DM}}$ versus $\omega_{\mathrm{B}}$ shows that there are at least two disjoint confidence regions in our seven dimensional space. These disjoint regions in Figure 5 correspond directly to the split confidence intervals observed in Figure 4 .

The disjoint regions observed in Figure 5, such as the plot of $\omega_{\mathrm{DM}}$ vs. $\omega_{\mathrm{B}}$, indicate that there are at least two disjoint confidence regions in the parameter space. These disjoint regions can also be seen in the $1 \mathrm{D}$ projections of $\omega_{\mathrm{DM}}, \omega_{\mathrm{B}}$, and $H_{0}$ shown in Figure 4 . We defer further discussion of the disjoint confidence regions to \$4.3. Smaller splits in the confidence intervals observed in nearly every plot in Figure 4 are a result of the fact that CMBFast does not return models which are perfectly continuous in the parameter space. While one may expect the derived confidence level to be smooth in parameter space, this 

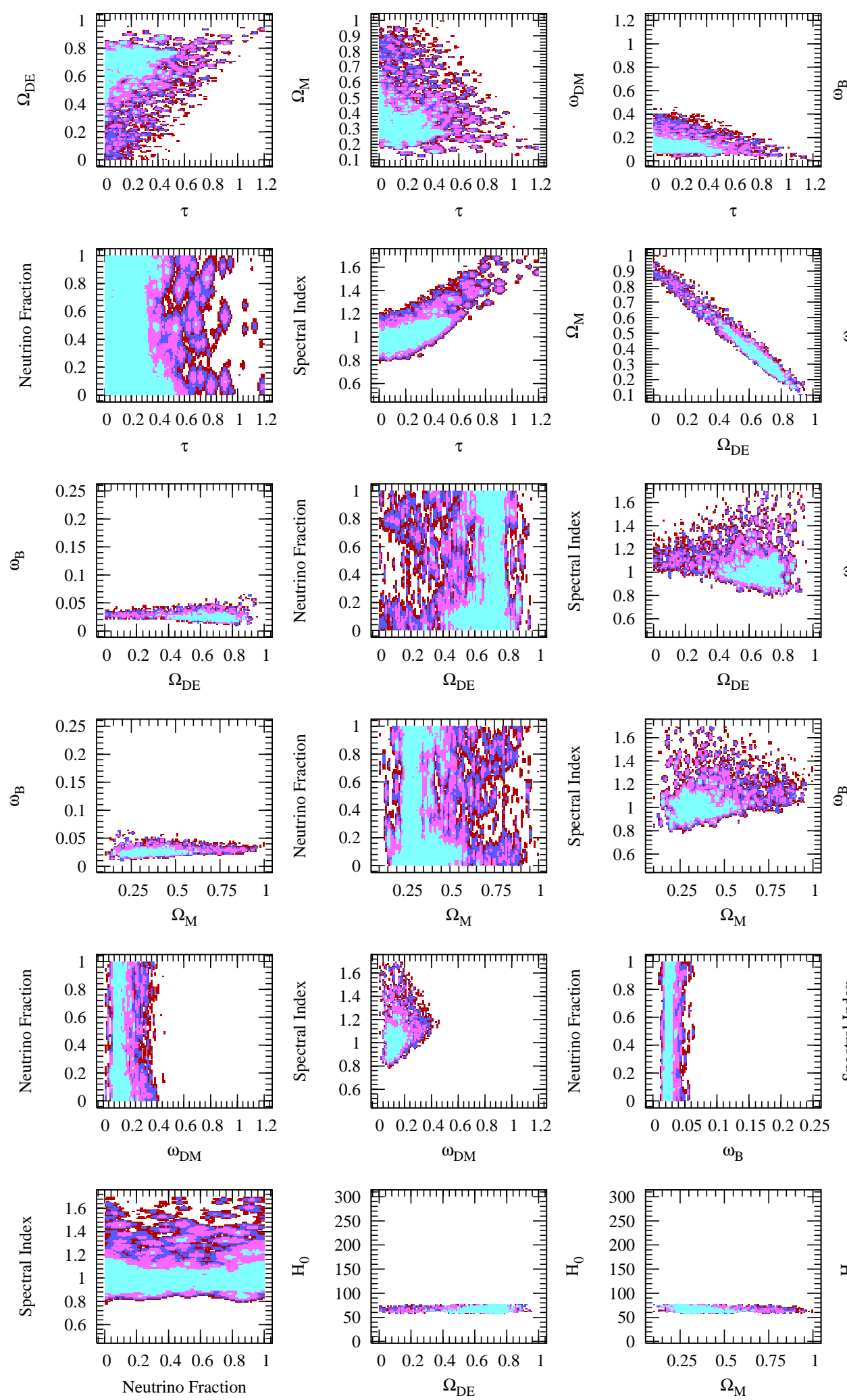
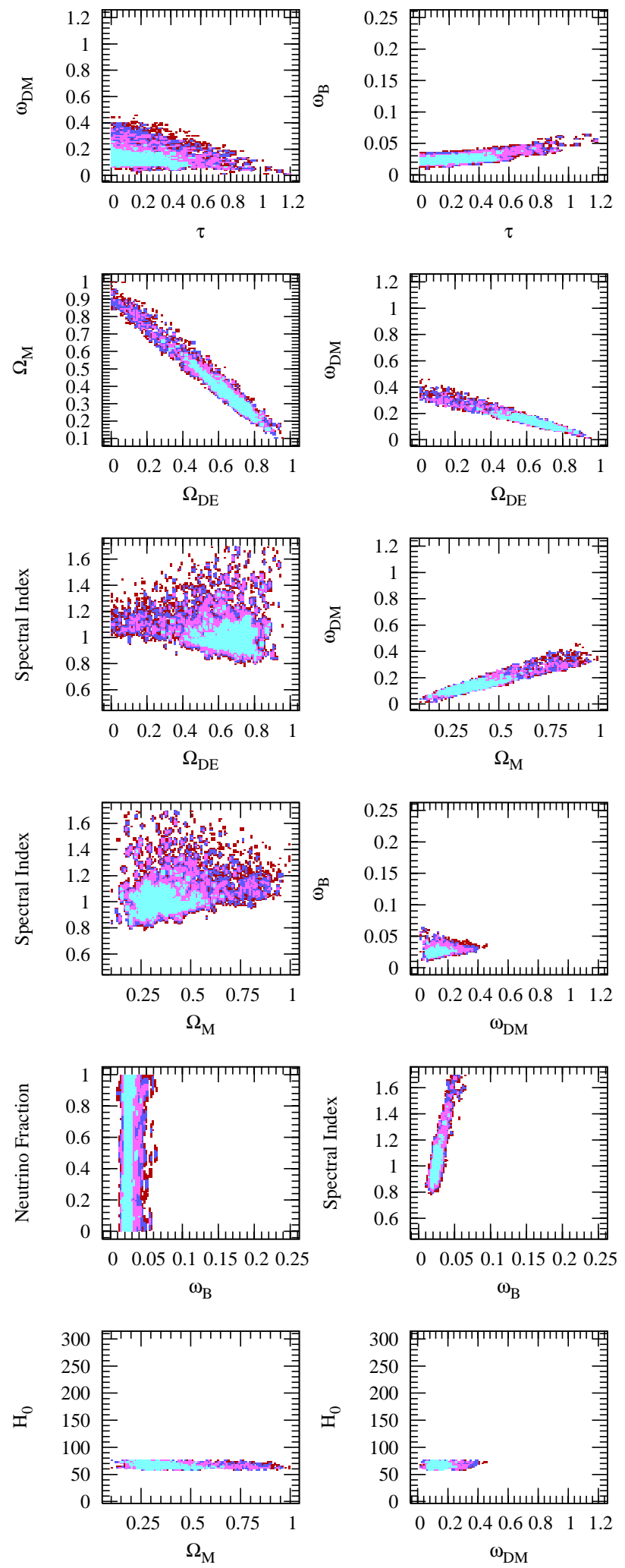

Fig. 7.- Jointly valid confidence regions for pairs of cosmological parameters, where we assume that that the value of $H_{0}$ is between 60 and $75 \frac{\mathrm{km} / \mathrm{s}}{\mathrm{Mpc}}$. The colors cyan, magenta, blue and red correspond to $\frac{1}{2} \sigma, \sigma, 1 \frac{1}{2} \sigma$ and $2 \sigma$, confidence levels, respectively. Areas of solid color indicate values for the given pair of fixed (plotted) parameters that contain the true value 
is not the case. We observe small discretizations and inconsistencies in the power spectrum model, which result in the confidence ball having a jagged, nebulous surface (as observed in Figure 5), rather than a perfectly smooth one. We will elaborate on this observation in 84.2 .

As illustrated in Figure 4, the confidence intervals for most parameters are not well constrained by the WMAP data alone. In particular, the constraint on the Hubble constant, $H_{0}$, is so weak as to allow values between 15 and 300 at the two sigma level; even at the one sigma level, $H_{0}$ ranges between 15 and 150 with additional fits at $H_{0} \sim 250$. The confidence intervals derived here cover the Bayesian credible intervals found in the literature using a variety of techniques (e.g. Tegmark et al. (2001); Spergel et al. (2003); Spergel et al. (2006)), as shown in Table 2, While the results in Table 2 are approximately centered on the same values, we are not in any way attempting to argue that the allowed parameter ranges are better, or worse, than those derived from alternative methods, as the comparison of credible (Bayesian) vs. valid (frequentist) parameter ranges is non-trivial and outside the scope of this work. A discussion of difference between the Bayesian and frequentist interpretations is given in $\$ 5.2$.

While this assessment may appear bleak, there is underlying structure to the confidence regions, hinted at by the disjoint regions in Figure 5. Suppose we restrict the range of a subset of our parameters and then compute the confidence intervals for the remaining parameters. Since our statistical model is independent of the ranges searched, we can compute these conditional confidence intervals without re-running any models. For any restriction of our parameter space, the confidence interval for a parameter of interest will be the union of the confidence intervals for that parameter over those confidence regions which obey our restriction. For example, in Figures 6 and 7 we show the effect on the confidence intervals and regions, respectively, of imposing the restriction that $H_{0}$ is between 60 and $75 \frac{\mathrm{km} / \mathrm{s}}{\mathrm{Mpc}}$. Note that with this restriction on $H_{0}$, the confidence intervals agree much better with the current estimate of the cosmological matter/energy budget and strongly suggest that $\Omega_{\text {Total }}=1$.

This analysis exhibits the power of our statistical inference technique: we can test constraints on one parameter, and see their effects on the remaining parameters without additional CMBFast computation or invalidation of statistical inferences. To this end, we have created a graphical interface that can be used to apply constraints and view the resulting effects in real time; this tool, along with the necessary data files, can be downloaded from http://gs3636.sp.cs.cmu.edu/visualizer/.

In the Bayesian view, the tightening of the allowable regions between Figures 4 and 6 and Figures 5 and 7 is analogous to what would occur when priors (either informative or noninformative) are applied. Such priors are universally applied in CMB cosmological analyses. As an example of how we can use this technique to better understand the cosmological 

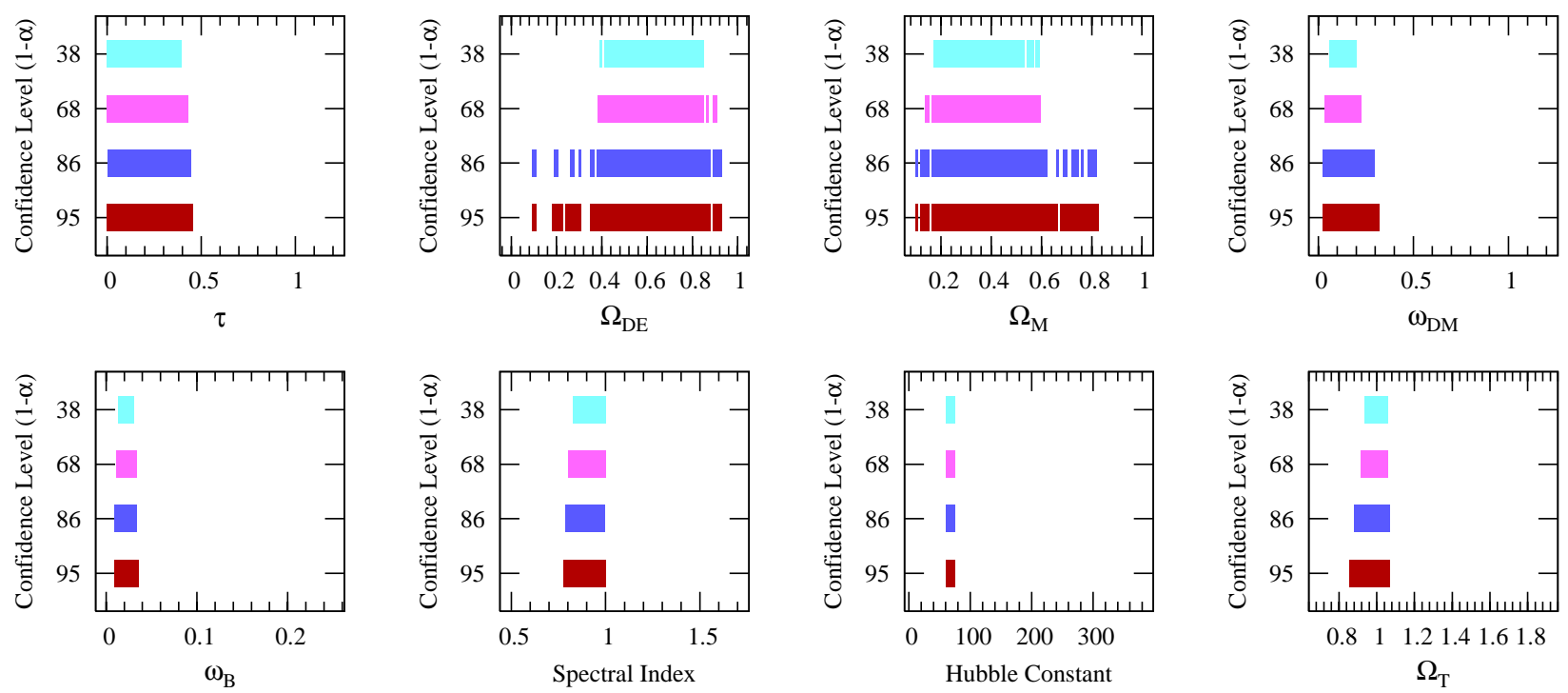

Fig. 8.- Jointly valid confidence intervals for our cosmological parameters, where we assume that $60 \frac{\mathrm{km} / \mathrm{s}}{\mathrm{Mpc}} \leq H_{0} \leq 75 \frac{\mathrm{km} / \mathrm{s}}{\mathrm{Mpc}}$ and $n_{s}<1$. Areas of solid color indicate values for the given parameter that contain the true value of cosmological parameter with probability $1-\alpha$, regardless of the values of the remaining 6 parameters. See the electronic edition of the Journal for a color version of this figure.

confidence surface, we focus in on one or two parameters and utilize the graphical interface described above.

WMAP Three Year data show that a scale invariant spectra $\left(n_{s}=1\right)$ is not a good fit to the WMAP Three Year data alone. If we place both the constraint that $n_{s}<1$ and that $60 \frac{\mathrm{km} / \mathrm{s}}{\mathrm{Mpc}} \leq H_{0} \leq 75 \frac{\mathrm{km} / \mathrm{s}}{\mathrm{Mpc}}$ on the WMAP One Year data, we see in Figure 8 that $\tau, \omega_{\mathrm{B}}$, and $\omega_{\mathrm{DM}}$ are much better constrained. More importantly, we see that the allowable ranges on $\omega_{\text {DM }}$ are forced into a single confidence range, in agreement with previous studies Spergel et al. $(2003)$.

Exploring the high $\omega_{\mathrm{DM}}$ space shown in Figure 4, we find that models consistent with high $\omega_{\mathrm{DM}}$ have large values of $\omega_{\mathrm{B}}(>0.05)$, as well as large Hubble constants $\left(>100 \frac{\mathrm{km} / \mathrm{s}}{\mathrm{Mpc}}\right)$. Both of these parameters are much better constrained in the WMAP Three Year data. This leads us to predict that the second confidence surface peak in the WMAP Three Year Data is less significant than in the WMAP One Year data (although this has yet to be shown). 


\subsection{Convergence}

Ideally, one would like to prove that our mapping from confidence ball radius to parameter space has converged. This could be done, for instance, by proving that our approximating model of spectrum distance as a function of cosmological parameters - that is our Gaussian process - has converged to the true values in those areas where the true values are near the radius of the $1-\alpha$ confidence ball. However, this effort has been confounded by a lack of continuity in the results returned by CMBFast. The method presented in this paper is not more susceptible to discontinuities than other techniques. Indeed, the convergence of most, if not all, inference methods will be adversely effected by the discontinuities of CMBFast models we observe in parameter space.

One standard assumption of function approximators is that of smoothness; that is that the underlying function to be modeled is continuous and differentiable. For Gaussian processes, this assumption motivates the usage of a covariance matrix in determining the relative weights of known samples when estimating values for unknown points. In this paper, we have also assumed that the covariance function is fixed over the entire space - that is that the underlying covariance is isotropic and homogeneous. These assumptions allow us to compute error bounds for each point in space, and enable us to determine when the model has converged to the underlying function.

However, experimentation shows that the underlying CMBFast function does not fulfill the continuous and differentiable assumptions, as shown in Figures 9 and 10, Both figures were produced by plotting the resulting model distance as we varied one parameter and kept the other six parameters fixed. Figure 9 shows a discretization effect that we believe is a result of integral approximations done by CMBFast. Discretization effects are common in simulated environments and it is reasonable to assume that the true function varies smoothly. More startling are the discontinuities revealed in Figure 10, Figure 10, shows that while on a broad scale the CMBFast function appears smooth, when one looks closer and closer, the function begins to act quite erratically. Of particular interest are the large discontinuity at $\Omega_{\mathrm{DE}}=0.446516$ and the seemingly random deviations from a smooth function throughout the entire range. These fluctuations in distance are not caused by random noise from CMBFast; CMBFast's output is deterministic given an input parameter vector.

There are two important implications of the results in Figures 9 and 10. First, we note that when parameter values result in spectra that are very close to the confidence ball radius, it is impossible to predict which side of the boundary a given point will be on, due to the inherent noise in CMBFast. For regions where many points are near the confidence ball radius, we will obtain spotty, jagged boundaries between those areas in the ball and those not. Secondly, the effects plotted in Figures 9 and 10 do not appear on the same range 


\begin{tabular}{|c|c|c|c|c|c|}
\hline Parameter & $\begin{array}{c}\text { No } \\
\text { Constraints }\end{array}$ & $60 \leq H_{0} \leq 75$ & $\begin{array}{c}n_{s}<1 \\
60 \leq H_{0} \leq 75\end{array}$ & $\begin{array}{c}\text { Spergel } \\
\text { et al. }(2003)\end{array}$ & $\begin{array}{c}\text { Spergel } \\
\text { et al. }(2006)\end{array}$ \\
\hline$\tau$ & $0-1.2$ & $0-0.94,1.17-1.2$ & $0-0.4$ & $\overline{0.095-0.242}$ & $0.058-0.117$ \\
\hline$\Omega_{\mathrm{DE}}$ & $0-0.94$ & $0-0.94$ & $0.39-0.9$ & & \\
\hline$\Omega_{\mathrm{M}}$ & $0-1.0$ & $0.13-0.95$ & $0.13-0.59$ & $0.22-0.36$ & $0.199-0.273$ \\
\hline$\omega_{\mathrm{DM}}$ & $0-0.36,0.62-0.70$ & $0.0-0.36$ & $0.03-0.2$ & & \\
\hline $100 \omega_{\mathrm{B}}$ & $0.5-6.2,11.5-12.7$ & $1.3-5.5$ & $1.3-3.2$ & $2.26-2.51$ & $2.15-2.31$ \\
\hline$f_{\nu}$ & $0-1$ & $0-1$ & $0-1$ & & \\
\hline$n_{s}$ & $0.73-1.7$ & $0.8-1.7$ & $0.84-1.0$ & $0.95-1.03$ & $0.944-0.978$ \\
\hline$\sigma_{8}$ & & & & $0.82-1.02$ & $0.71-0.81$ \\
\hline$H_{0}$ & $17-135,243-272$ & $60-75$ & $60-75$ & $67-77$ & $70.3-76.7$ \\
\hline
\end{tabular}

Table 2: Derived $68 \%$ confidence intervals. Those to the left of the solid line are derived from Figures 4, 6] and 8 respectively, while those to the right are quoted from referenced literature.

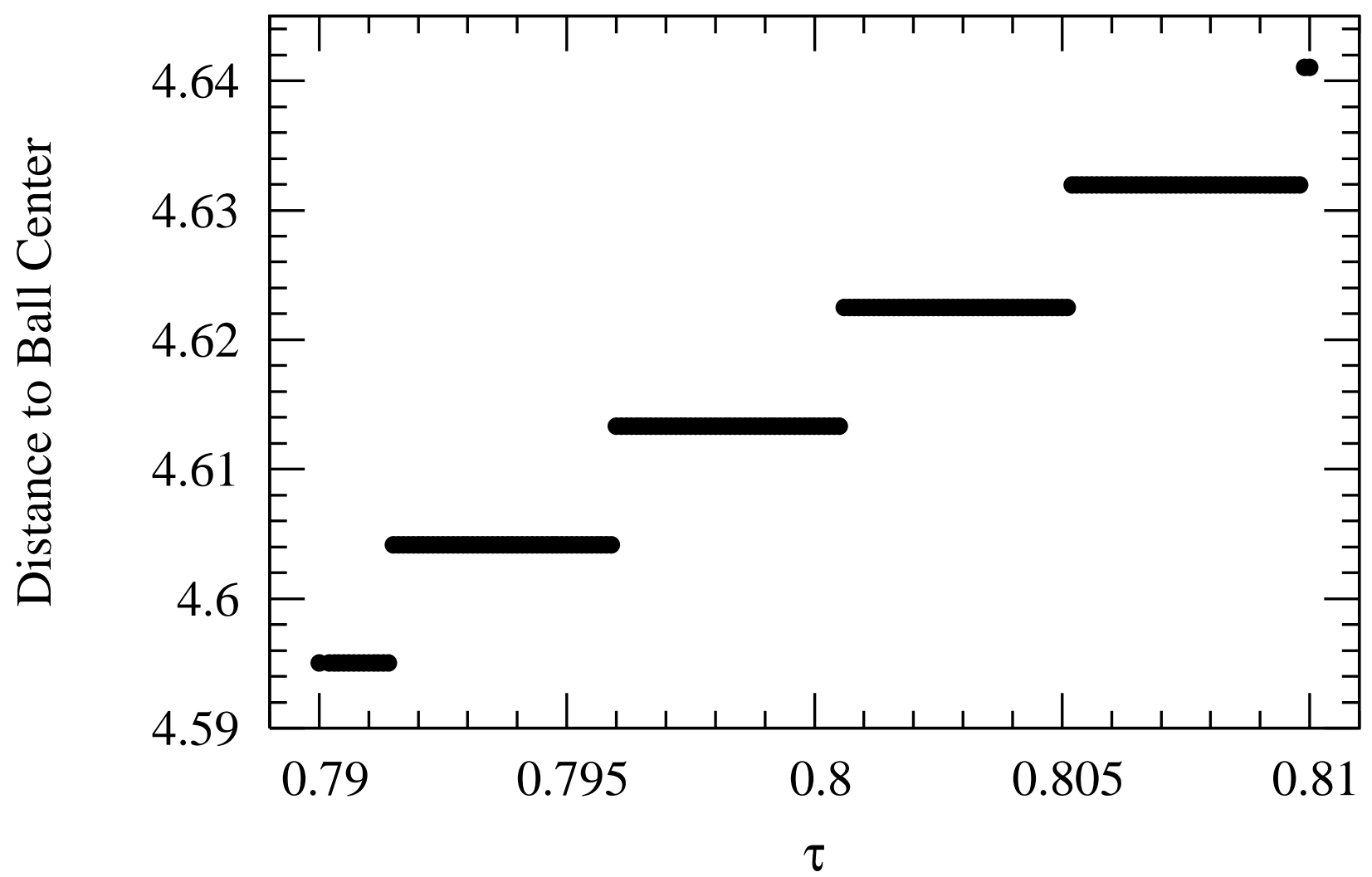

Fig. 9.- A plot of spectra distance as a function of $\tau$, with all other parameters fixed, showing the discretization of CMBFast. For these experiments $\vec{x}=\left\{\tau, \Omega_{\mathrm{DE}}, \Omega_{\mathrm{M}}, \omega_{\mathrm{DM}}, \omega_{\mathrm{B}}, f_{\nu}, n_{s}\right\}$ $=\{\tau, 0.0,0.2,0.8,0.003,0.0,1.2\}$. 
scales. This makes it more difficult to determine the correct level of smoothing, and hence discover the true underlying function. Thus, while it is still possible to deduce approximate covariances among the variables, it becomes impossible to ensure the model has correctly converged to the true model.

We note that this lack of continuity will adversely effect the convergence of any model that relies on the smoothness of the underlying function, be it MCMC or Gaussian processes. In the case of MCMC, the discontinuities in the variance weighted sum of squares between the models computed by CMBFast and the data require that comprehensive sampling of the posterior be performed to ensure that the peaks and valleys in any local region are correctly averaged out, ensuring that the integral over the posterior is correctly computed. While we can run both methods in a mode that smooths over these discontinuities (by effectively ignoring them), we must realize that the resulting algorithms will converge to a solution that is incorrect. Additionally, increasing the sampling of either algorithm would eventually turn up the existence of these discontinuities, and the system would jump from an apparent convergence in the smoothed case, to a new convergence where discontinuities are considered. We elaborate on this idea further in $\$ 5.2$.

\subsection{Connectivity}

As Figure 5 shows, there are two main peaks that lie above the $1 \sigma$ confidence ball radius. As a test of the function approximator's convergence, we conducted focused tests to see if these peaks were truly connected. In particular, we used the semi-variance matrix of the Gaussian process to compute the maximal influence distance from a given point one could travel before possibly encountering the $1-\alpha$ confidence ball radius. We then created clusters of points above the $68 \%$ confidence ball radius using a friends-of-friends algorithm; that is, a point is added to an existing group if it is within the maximal influence distance of any point currently in the group. Starting with all points in their own groups, we first passed through the data, merging groups where possible. Then, additional points were sampled between existing groups, using an $\mathrm{A}^{*}$ like algorithm (Hart et al. 1968). For two groups $A$ and $B$, we found the point, $x$, in $A$ that was closest to any point in $B$. We then created a set of candidate points within the influence distance of $x$, and add them to a queue, $\mathcal{Q}$, sorted according to their distances to $B$. We then take the point $p$ from $\mathcal{Q}$ that is closest to $B$ run it through CMBFast and compare to our confidence ball. If $p$ is within our confidence radius, then we create candidate points for $p$ (just as we did for $x$ ) and add them to $\mathcal{Q}$. Otherwise, we remove $p$ from $\mathcal{Q}$. This procedure is repeated until either $B$ is within the influence distance of $p$ or we exhaust $\mathcal{Q}$. 
The primary data set contained roughly 2000 distinct groups, which were quickly merged using the friends-of-friends algorithm. This left us with 2 major clusters shown in Figure

5. Using the algorithm noted above, we were unable to find connections between the main peak and the secondary peak, even after multiple attempts starting from different locations. We believe that there exists no smooth transition of variable parameters that leads from the concordance to the secondary peak. The second peak is not just an extension of the concordance peak that appears disjoint due to under sampling or projection effects.

\section{Comparison to Alternative Methods of Statistical Inference}

In $\S_{4}$, we showed that the results of our technique are quite similar to other statistical inference methods currently employed in the literature. Let us now relate our method to other inference techniques, and point out a few subtle, but remarkable, distinctions between them.

\section{1. $\chi^{2}$ Tests}

The method presented in $\$ 2.2$ can be succinctly described as a method which computes the weighted sum of squares of the regressed fit and the test spectrum at the data points and rejects the hypothesis that the test spectrum could be generated by the data if the weighted sum is greater than the constant given in Equation 3. Intuitively, this process is quite similar to using a $\chi^{2}$ test, with two important differences.

First, our technique is centered around a nonparametric fit to the data, not the data themselves. As a result, our method is approximately centered on the true underlying function, $f$, as opposed to the noisy observations of $f$. The implication is that our method is less affected by noise in the data, than simple $\chi^{2}$ tests. In particular, we have observed that $\chi^{2}$ tests will reject all models in cases where there is a single outlier $4 \sigma$ from the maximum likelihood estimate fit. By initially fitting a nonparametric function to the data and then using this function to compute sum-of-squares distances, we are much less susceptible errors caused by noisy outliers.

Secondly, the radius computed using the pivot process is smaller than the $\chi^{2}$ radius, as we consider the Gaussian errors of all points as an ensemble, not individually as with $\chi^{2}$ tests. The smaller radius of the pivot process translates directly into smaller confidence regions as compared with those found using $\chi^{2}$ tests. This allows us to reject more of the hypothesis test models, and subsequently return tighter bounds on the parameters of interest. The 
confidence ball test has more statistical power than does the $\chi^{2}$ test. A comparison of the relative widths of the confidence and $\chi^{2}$ balls is shown in Figure 11,

\subsection{Bayesian Techniques}

As noted in $\$ 1$ most $\mathrm{CMB}$ power spectrum parameter estimations to date have been done via Bayesian techniques (e.g., Knox et al. (2001); Gupta \& Heavens (2002); Spergel et al. (2003); Jimenez et al. (2004); Dunkley et al. (2005)). Since the prior distribution is not conjugate on the likelihood, computing the posterior involves estimating an integral over the entire space spanned by the prior. Perhaps the most straight-forward way to compute this integral is with an evenly-spaced grid with $n$ points per parameter. For this approach, one pre-specifies a $d$-dimensional grid (where $d$ is the number of parameters of interest) and computes the posterior at the center of each grid cell. The integral is then (approximately) the sum of the posterior at each grid cell, and the $1-\alpha$ credible intervals can be determined (usually by marginalization) to be the smallest range for a given parameter that contains $1-\alpha$ of the posterior probability. While straight forward, this approach scales exponentially with dimension, and hence is infeasible for even moderate dimensions; we estimate that a grid based approach, using CMBFast and seven parameters (similar to our method), with just 10 grid spacings per parameter would take over 100 years on a single computer.

As a result of the dimensionality problem, Markov Chain Monte Carlo (MCMC) has become an increasingly popular approach for estimating posteriors due to their (perceived) computational efficiency (e.g Gupta \& Heavens (2002); Jimenez et al. (2004); Sandvik et al. (2004); Dunkley et al. (2005); Chu \& Knox (2005)). In the MCMC technique, new samples are often derived using the Metropolis-Hastings algorithm. The Metropolis-Hastings algorithm chooses a new sample $x$ from some arbitrary (pre-specified) proposal distribution defined over the $d$-dimensional parameter space based on the previous sample and then accepts or rejects $x$ based on the ratio of the proposed and current posterior density (when the proposal distribution is symmetric, as is common). The algorithm samples the input space roughly in proportion to the expected probability of each location.

Theoretically MCMC using Metropolis-Hastings algorithm converges almost surely to the stationary distribution (the posterior) in the limit of infinite sampling. However, it is quite difficult to determine if convergence has been met with a finite number samples. In particular, if a posterior is comprised by two narrow, spatially separated Gaussians, then the probability of transition from one Gaussian to the other will be vanishingly small. Thus, after the chain has rattled around in one of the peaks for a while, it will appear that the chain has converged; however, after some finite amount of time, the chain will 
suddenly jump to the other peak, revealing that the initial indications of convergence were incorrect. As this example illustrates, if the Markov chain is run with too few examples, the resulting credible intervals will be too narrow, and thus will not truly contain $1-\alpha$ of the probability mass. Thus, the consequence of lack of true convergence is artificially small credible intervals. This problem is usually skirted by assuming that there are no small isolated peaks, computing multiple independent chains and comparing the results to illustrate convergence. Additionally, Dunkley et al. (2005) and others have proposed alternative methods to detect convergence. However, none of these methods are able to prove convergence with a limited number of CMBFast runs.

Moreover, as we noted in $₫ \mathbb{1}, \mathrm{MCMC}$ is designed to draw samples from an unknown distribution, not to search that distribution. As a result, MCMC algorithms explicitly spend a large number of samples on high-likelihood regions, and a minimal number on low-likelihood regions. However, when we are computing $1-\alpha$ confidence intervals, it is the low-likelihood regions (those around the $1-\alpha$ boundary) that we are interested in. In contrast, a search algorithm that can directly look up the likelihood of a sample has no reason to spend a large number of samples near the peak of the distribution, and can instead focus on the boundary in question.

These differences are clearly shown in Figure 12, which depicts (with black dots) samples chosen by typical single runs of MCMC and our algorithm when trying to compute the $95 \%$ credible/confidence intervals for a standard normal distribution 2. Both algorithms were constrained to samples chosen in $[-10: 10]$. The MCMC algorithm was started at a randomly selected point, with a uniform prior over the range. In this figure we use a standard normal proposal distribution, although the sampling pattern is similar for other distributions we tried. Credible intervals for MCMC and confidence intervals for our algorithm are depicted below the plots. Several points are quite apparent. First MCMC has failed to converge in 50 samples, while our algorithm has converged nicely. The credible intervals given by MCMC are not only underestimated, but are also not centered on the true distribution's center, revealing a potential liability for interpreting MCMC chains which have not converged.

Secondly, notice that MCMC heavily samples the peak of the distribution, while our algorithm focus on those regions associated with the confidence interval boundaries. The MCMC chain results in a ragged collection of disjoint credible intervals, while our algorithm returns a single interval in which the endpoints have been well determined.

\footnotetext{
${ }^{2}$ For the Bayesian case, we assume that the observed data is a single point at the origin. As a result, the true posterior derived via sampling will be exactly the same as the true standard Normal distribution. This is done to ensure that both algorithms are sampling the same function, allowing us to compare the sampling patterns of the algorithms.
} 
Thirdly, note that our algorithm samples extreme points to ensure that it has not failed to observe additional peaks in the distribution which may contribute to the $95 \%$ confidence interval, while MCMC has not. As noted before, since MCMC is not a search algorithm, it may spend a large number of samples in a single distribution peak before jumping to another peak in the distribution. This sampling pattern may cause MCMC to appear to have converged, when in reality it has just failed to transition to the second peak, as in the two Gaussian case described previously.

Finally, we note that the MCMC algorithm is not data efficient. While Figure 12 depicts those experiments run by MCMC, the final MCMC chain consists of only those points that were accepted (in this case by the Metropolis-Hastings algorithm). As such, some of the points that MCMC samples are discarded immediately, and never used to guide the chain in future steps, or to determine the $1-\alpha$ credible intervals. In addition, many MCMC practitioners remove all but every $j$ th sample point (for some integer $j$ ) to ensure that the points in the chain are truly independent. This significantly reduces data efficiency.

\subsection{Advantages of Frequentist Inference}

Often, non-statisticians are confused by differences between Bayesian and frequentist techniques, and the advantages and limitations that each maintains. Particularly appealing with the Bayesian approach is the fact that one is computing a posterior distribution over the parameter space. Thus, not only does one obtain $1-\alpha$ credible intervals, but one

gets a sense of where within the interval, the true value is expected to be. Frequentist approaches do not allow for one to compute the probability that the true value is equal to some particular parameter value. While choosing one technique over the other is a matter of personal statistical philosophy, we believe that frequentist approaches hold important advantages over their Bayesian counterparts.

First, any Bayesian technique requires that one assume a family of likelihood functions and a prior distribution over the parameter space in order to compute the posterior. The resulting posterior is only as valid as both the likelihood and the prior. In many cases, a prior distribution is unknown. In these cases, an "uninformative prior," equivalent to a uniform distribution on some bounded range, is often assumed. However, such a prior is not uninformative. In particular, a uniform prior indicates that the practitioner believes that the true distribution of the parameter is uniform, not unknown. Moreover "uninformative" priors are parametrization dependent. If we reformulate our 7D CMB problem by replacing $\Omega_{M}$ with $H_{0}$, a uniform prior over the original problem will not translate into a uniform prior over the formulation including $H_{0}$, as $\Omega_{M}$ is inversely related to $H_{0}$. 
Secondly, any change to the prior invalidates the current results. In particular, even when one is using a uniform prior, merely changing parameter ranges will result in a different posterior with possibly different $1-\alpha$ credible intervals. Thus analyses, like those we performed in 4.1 would have required us to recompute the entire chain (or set of chains), an extremely expensive proposition, or somehow approximate the difference. Additionally, for Bayesian techniques, the prior should be independent of the data, and hence it should not be changed after observing the data. By recomputing the posterior using a new prior (based upon a previous posterior), we open ourselves to errors incurred due to multiple hypothesis testing. Moreover, it is a small step from such repeated Bayesian inferences to data-dependent priors, which are incoherent not Bayesian. Hence, data-dependent priors do not benefit from theoretical guarantees derived for Bayesian analyses, which assume priors are chosen before any data is observed.

It is interesting to note that Table 1 denotes the final ranges of parameters searched. We initially started with the same parameter ranges as (Tegmark et al. 2001), but increased our ranges slightly to better capture a secondary peak in confidence space (shown in Figure 5). Because of our frequentist based technique, we can easily change the ranges being searched without re-running any of the CMBFast models, or recomputing any of our current inferences. This contrasts sharply with Bayesian techniques.

Finally, recall from $\$ 1$ that Bayesian approaches answer a fundamentally different question than do frequentist approaches. Frequentist approaches are concerned with deriving procedures which will return confidence intervals that trap the true value of a parameter in at least $1-\alpha$ of the cases in which the procedure is used. Bayesian methods are more interested in determining the probability that a particular value of a parameter is chosen for the given data set and prior. While we can compute "credible" intervals for Bayesian methods by choosing the minimum range of a parameter such that the enclosed probability is equal to $1-\alpha$, these intervals do not necessary correspond to those derived from using a frequentist approach. In particular, there is no guarantee that credible intervals will contain the true value of the parameter in at least $1-\alpha$ fraction of the instances where the technique is applied. Specifically, when the likelihood function of the model goes awry, such as in cases of high-dimension, missing data, and/or non-parametric models, the inference made using Bayesian methods will be incorrect.

This problem is particularly acute for high dimensions, where $1-\alpha$ credible intervals might trap the true value of the parameter close to zero percent of the time. That is, if Bayesian techniques are applied to a series of data sets, the fraction of the resulting $1-\alpha$ credible intervals that contain the true values of the parameter will be less than $1-\alpha$ and may be significantly less that $1-\alpha$. While we find this fact disturbing, a Bayesian 
might be willing to trade off the fact that the credible intervals usually will not contain the truth for the ability to compute a posterior distribution of likelihood over parameter space (assuming some prior) and hence determine the probability of any given parameter setting. As, Wasserman (2004) notes: "to construct procedures with guaranteed long run performance, such as confidence intervals, use frequentist methods."

\section{Conclusions}

In this paper, we present a new technique to map confidence surfaces, and show results on first-year WMAP data. This method, utilizing a non-parametric fit and confidence balls, allows for computing simultaneously valid confidence intervals. Our technique is similar in spirit to the Bayesian methods, but differs significantly in that it is a frequentist analysis with simultaneous valid coverage. Thus, the derived confidence intervals are valid regardless of the values of the remaining parameters. This is not the case when a maximization or marginalization technique is used. While the use of confidence balls requires a search over the entire parameter space akin to the integration required for Bayesian techniques, we present an algorithm to efficiently compute regions of parameter space which have confidence values above a specified $1-\alpha$ threshold. We present results of our algorithm and note that they are similar to those derived using alternative statistical methods. While the WMAP power spectrum data alone is insufficient to constrain any of the cosmological parameters, the addition of a reasonable assumption on the Hubble constant, provides useful cosmological insights.

We point out that the purpose of this paper is to present a new statistical and computational technique to provide frequentist confidence intervals on the cosmological parameters using the WMAP Year 1 data. We are not arguing that the allowed parameter ranges shown in Figures 4, 5, 6 and 7 are more accurate than those presented by the WMAP team. The reason for this is two-fold: (1) the comparison of credible (Bayesian) vs. valid (frequentist) parameter ranges is non-trivial and outside the scope of this work and (2) we use only the WMAP Year 1 data, while others have utilized non-WMAP data in various ways to provide additional constraints on the parameters.

Analysis of Figures 4 and 5 shows that the one sigma confidence regions are similar to those found in the literature using a variety of techniques (e.g. Tegmark et al. (2001); Spergel et al. (2003); Spergel et al. (2006)). Figures 4 and 5 illustrate that the WMAP data alone is not sufficient to strongly constrain the matter/energy budget for the Universe. In particular, the constraint on the Hubble constant, $H_{0}$, is so weak as to allow values between 15 and 300 at the two sigma level. 
If we instead constrain $H_{0}$ to a more "typical" range of [60:75], we get much tighter constraints on all parameters, as shown in Figures 6 and 7 . Because we are using a frequentist confidence procedure, adding the restriction does not affect the validity of the inference. Moreover, no additional CMBFast models must be computed to test this constraint, illustrating the power of our statistical procedure. Note that both Figures 6] and 7 agree much better with the current estimates of the cosmological matter/energy budget and strongly suggest that $\Omega_{\text {Total }}=1$.

Moreover, as we show in 4 4.2. CMBFast creates temperature power spectra which are discontinuous in parameter space. This discontinuity violates the smoothness assumption of the underlying target function used by both our Gaussian process technique, as well as by MCMC. This makes convergence statements difficult to make. However, we believe that the 1.2 million models run show reasonable convergence. We believe that with additional assumptions on CMBFast — such as the maximum size of a discontinuity — we will be able to prove that our method converges in a reasonable time frame.

Additionally, we show that comparing CMBFast models to the WMAP year 1 temperate power spectrum data results in a multi-modal solution in confidence space. We have detected at least two distinct confidence regions in parameter space. However, by adding assumptions on $n_{s}$, we can eliminate the secondary peak, leading us to believe that the secondary peak may not be visible in the WMAP third year data.

In summary, we believe the proposed approach of using a non-parametric fit to the data and confidence balls, coupled with a search algorithm to find models in parameter space which fit our regressed estimate, provides a robust and informative method for computing confidence intervals for cosmological parameters. In addition to merely computing intervals, our approach has the ability to test various constraints without computing new models or making assumptions about which models should be fit and what the ranges of the parameter space should be. We are working on techniques to prove convergence of the algorithm, as well as the incorporation of additional data sets to further constrain the mass/energy budget of the Universe.

The authors would like to thank the referee for his/her valuable suggestions and corrections.

Facilities: WMAP 


\section{A. Estimating $\tau$}

Recall from $\$ 2.2 .1$ that the cosine basis is defined on $[0,1]$ by

$$
\phi_{j}(x)= \begin{cases}1 & \text { for } j=0 \\ \sqrt{2} \cos (\pi j x) & \text { for } j=1,2,3, \ldots\end{cases}
$$

If $j$ and $k$ are distinct, positive integers, then

$$
\begin{aligned}
\phi_{j} \phi_{k} & =2 \cos (\pi j x) \cos (\pi k x) \\
& =\cos (\pi(j+k) x)+\cos (\pi(j-k) x) \\
& =\frac{1}{\sqrt{2}}\left(\phi_{j+k}+\phi_{|j-k|}\right) .
\end{aligned}
$$

Moreover, if $j>0$, then $\phi_{j}^{2}=2 \cos ^{2}(\pi j x)=\cos (2 \pi j x)+1=\frac{1}{\sqrt{2}} \phi_{2 j}+\phi_{0}$. Therefore, as mentioned in $\$ 2.2 .1$,

$$
\Delta_{j k \ell}=\left\{\begin{array}{cl}
1 & \text { if } \#\{j, k, l=0\}=3 \\
0 & \text { if } \#\{j, k, l=0\}=2 \\
\delta_{j k} \delta_{0 \ell}+\delta_{j \ell} \delta_{0 k}+\delta_{k \ell} \delta_{0 j} & \text { if } \#\{j, k, l=0\}=1 \\
\frac{1}{\sqrt{2}}\left(\delta_{\ell, j+k}+\delta_{\ell,|j-k|}\right) & \text { if } \#\{j, k, l=0\}=0
\end{array} .\right.
$$

Let $w(x)=1 / \sigma^{2}(x)$, such that $w^{2}(x)=\sum_{j} w_{j} \phi_{j}(x)$. As in 92.2 .1 , we let $\hat{\mu}_{j}=\lambda_{j} Z_{j}$, where

$$
Z_{j}=\frac{1}{n} \sum_{i=1}^{n} Y_{i} \phi_{j}\left(X_{i}\right)
$$

and $1 \geq \lambda_{0} \geq \lambda_{1} \geq \cdots \geq \lambda_{n} \geq 0$ are shrinkage coefficients. In this work, we use a special case of monotone shrinkage in which

$$
\lambda_{j}= \begin{cases}1 & \text { for } j \leq J \\ 0 & \text { for } j>J\end{cases}
$$

for $J \in[0,1,2, \ldots, n]$ such that $J$ minimizes Stein's unbiased risk estimate given in Equation 2. With these definitions, the loss can be written as

$$
\begin{aligned}
L(f, \hat{f}) & =\int_{0}^{1}\left(\frac{\hat{f}(x)-f(x)}{\sigma(x)}\right)^{2} d x \\
& =\sum_{j, k, \ell}\left(\mu_{j}-\hat{\mu}_{j}\right)\left(\mu_{k}-\hat{\mu}_{k}\right) w_{\ell} \int_{0}^{1} \phi_{j} \phi_{k} \phi_{\ell} \\
& =\sum_{j, k}\left(\mu_{j}-\hat{\mu}_{j}\right)\left(\mu_{k}-\hat{\mu}_{k}\right) \sum_{\ell} w_{\ell} \Delta_{j k \ell} \\
& =(\mu-\hat{\mu})^{T} W(\mu-\hat{\mu}),
\end{aligned}
$$


where $W_{j k}=\sum_{\ell} w_{\ell} \Delta_{j k \ell}$. As in \$2.2.1, let $D$ and $\bar{D}=1-D$ be diagonal matrices with 1's in the first $J$ and last $n-J$ entries respectively. Then $\hat{\mu}=D Z$, where $Z$ is again assumed to be Normal $(\mu, B)$. Thus, $\mathrm{E}[\hat{\mu}]=D \mu, \operatorname{Cov}\left(\hat{\mu}_{j}, \hat{\mu}_{k}\right)=\lambda_{j} \lambda_{k} B_{j k}$ and $\operatorname{Var}(\hat{\mu})=D B D$. The risk then becomes

$$
\begin{aligned}
R=\mathrm{E}[L] & =\mathrm{E}\left[(\mu-\hat{\mu})^{T} W(\mu-\hat{\mu})\right] \\
& =\operatorname{trace}(D W D B)+\mu^{T} \bar{D} W \bar{D} \mu \\
& =\operatorname{trace}(D W D B)+\sum_{j, k} \mu_{j} \mu_{k} \bar{\lambda}_{j} \bar{\lambda}_{k} W_{j k}
\end{aligned}
$$

An unbiased estimate can be obtained by replacing $\mu_{j} \mu_{k}$ with $Z_{j} Z_{k}-B_{j k}$. The result is

$$
\hat{R}=Z^{T} \bar{D} W \bar{D} Z+\operatorname{trace}(D W D B)-\operatorname{trace}(\bar{D} W \bar{D} B)
$$

It follows that

$$
\hat{L}-\hat{R}=\mu^{T} W \mu-Z^{T} C+Z^{T} A Z+\operatorname{trace}(A Z)
$$

where $A=D W+W D-W$ and $C=2 D W \mu$. Moreover,

$$
\begin{aligned}
\operatorname{Var}(\hat{L}-\hat{R}) & =\operatorname{Var}\left(Z^{T} A Z-Z^{T} C\right) \\
& =\operatorname{Var}\left(Z^{T} A Z\right)+\operatorname{Var}\left(Z^{T} C\right)-2 \operatorname{Cov}\left(Z^{T} A Z, Z^{T} C\right) \\
& =2 \operatorname{trace}(A B A B)+\mu^{T} Q \mu
\end{aligned}
$$

where $Q=A B A+W D B D W-2 A B D W$. Plugging in unbiased estimates of the linear and quadratic forms involving $\mu$, we get the following estimate for the variance of the pivot process:

$$
\hat{\tau}^{2}=2 \operatorname{trace}(A B A B)+Z^{T} Q Z-\operatorname{trace}(Q B) .
$$

\section{REFERENCES}

Abroe, M. E., Balbi, A., Borrill, J., Bunn, E. F., Hanany, S., Ferreira, P. G., Jaffe, A. H., Lee, A. T., Olive, K. A., Rabii, B., Richards, P. L., Smoot, G. F., Stompor, R., Winant, C. D., \& Wu, J. H. P. 2002, MNRAS, 334, 11

Bennett, C. L., Hill, R. S., Hinshaw, G., Nolta, M. R., Odegard, N., Page, L., Spergel, D. N., Weiland, J. L., Wright, E. L., Halpern, M., Jarosik, N., Kogut, A., Limon, M., Meyer, S. S., Tucker, G. S., \& Wollack, E. 2003, ApJS, 148, 97

Beran, R. 2000, Journal of the American Statistical Association, 95, 155

Beran, R. \& Dümbgen, L. 1998, Annuals of Statistics, 26, 1826 
Bryan, B., Schneider, J., Nichol, R. C., Miller, C. J., Genovese, C. R., \& Wasserman, L. 2005, in Advances in Neural Information Processing Systems 18 (Cambridge, MA: MIT Press)

Christensen, N., Meyer, R., Knox, L., \& Luey, B. 2001, Classical and Quantum Gravity, 18, 2677

Chu, M. \& Knox, L. 2005, ApJ, 620, 1

Cressie, N. A. C. 1991, Statistics for Spatial Data (New York: Wiley)

Dunkley, J., Bucher, M., Ferreira, P. G., Moodley, K., \& Skordis, C. 2005, MNRAS, 356, 925

Fendt, W. A. \& Wandelt, B. D. 2006, ArXiv Astrophysics e-prints

Genovese, C., Miller, C. J., Nichol, R. C., Arjunwadkar, M., \& Wasserman, L. 2004, Statistic Science, 19, 308

Gorski, K. M., Stompor, R., \& Juszkiewicz, R. 1993, ApJ, 410, L1

Griffiths, L. M., Silk, J., \& Zaroubi, S. 2001, MNRAS, 324, 712

Guestrin, C., Krause, A., \& Singh, A. P. 2005, in ICML '05: Proceedings of the 22nd international conference on Machine learning (New York, NY, USA: ACM Press), $265-272$

Gupta, S. \& Heavens, A. F. 2002, MNRAS, 334, 167

Hajian, A. 2006, ArXiv Astrophysics e-prints

Hart, P., Nilsson, N., \& Raphael, B. 1968, in IEEE Trans. on Systems Science and Cybernetics, Vol. 4, IEEE, 100-107

Hinshaw, G., Spergel, D. N., Verde, L., Hill, R. S., Meyer, S. S., Barnes, C., Bennett, C. L., Halpern, M., Jarosik, N., Kogut, A., Komatsu, E., Limon, M., Page, L., Tucker, G. S., Weiland, J. L., Wollack, E., \& Wright, E. L. 2003, ApJS, 148, 135

Jimenez, R., Verde, L., Peiris, H., \& Kosowsky, A. 2004, Phys. Rev. D, 70, 023005

Kaplinghat, M., Knox, L., \& Skordis, C. 2002, ApJ, 578, 665

Knox, L., Christensen, N., \& Skordis, C. 2001, ApJ, 563, L95

Lewis, A. \& Bridle, S. 2002, Phys. Rev. D, 66, 103511 
Lewis, A., Challinor, A., \& Lasenby, A. 2000, ApJ, 538, 473

MacKay, D. 1992, Neural Computation, 4, 590

Miller, C. J., Nichol, R. C., Genovese, C., \& Wasserman, L. 2001, Bulletin of the American Astronomical Society, 33, 1358

Padmanabhan, T. \& Sethi, S. K. 2001, ApJ, 555, 125

Sandvik, H. B., Tegmark, M., Wang, X., \& Zaldarriaga, M. 2004, Phys. Rev. D, 69, 063005

Schafer, C. M. \& Stark, P. B. 2003, in Proceedings for Statistical Problems in Particle Physics, Astrophysics, and Cosmology, SLAC

Seljak, U. \& Zaldarriaga, M. 1996, Astrophyical Journal, 469, 437

Seljak, U. c. v., Sugiyama, N., White, M., \& Zaldarriaga, M. 2003, Phys. Rev. D, 68, 083507

Spergel, D. N., Bean, R., Dore, O., Nolta, M. R., Bennett, C. L., Hinshaw, G., Jarosik, N., Komatsu, E., Page, L., Peirisand, H. V., Verde, L., Barnes, C., Halpern, M., Hill, R. S., Kogut, A., Limon, M., Meyer, S. S., Odegard, N., Tucker, G. S., Weiland, J. L., Wollack, E., \& Wright, E. L. 2006, astro-ph/0603449

Spergel, D. N., Verde, L., Peiris, H. V., Komatsu, E., Nolta, M. R., Bennett, C. L., Halpern, M., Hinshaw, G., Jarosik, N., Kogut, A., Limon, M., Meyer, S. S., Page, L., Tucker, G. S., Weiland, J. L., Wollack, E., \& Wright, E. L. 2003, ApJS, 148, 175

Tegmark, M. 1999, ApJ, 514, L69

Tegmark, M., Strauss, M. A., Blanton, M. R., Abazajian, K., Dodelson, S., Sandvik, H., Wang, X., Weinberg, D. H., Zehavi, I., Bahcall, N. A., Hoyle, F., Schlegel, D., Scoccimarro, R., Vogeley, M. S., Berlind, A., Budavari, T., Connolly, A., Eisenstein, D. J., Finkbeiner, D., Frieman, J. A., Gunn, J. E., Hui, L., Jain, B., Johnston, D., Kent, S., Lin, H., Nakajima, R., Nichol, R. C., Ostriker, J. P., Pope, A., Scranton, R., Seljak, U., Sheth, R. K., Stebbins, A., Szalay, A. S., Szapudi, I., Xu, Y., Annis, J., Brinkmann, J., Burles, S., Castander, F. J., Csabai, I., Loveday, J., Doi, M., Fukugita, M., Gillespie, B., Hennessy, G., Hogg, D. W., Ivezić, Ž., Knapp, G. R., Lamb, D. Q., Lee, B. C., Lupton, R. H., McKay, T. A., Kunszt, P., Munn, J. A., O’Connell, L., Peoples, J., Pier, J. R., Richmond, M., Rockosi, C., Schneider, D. P., Stoughton, C., Tucker, D. L., vanden Berk, D. E., Yanny, B., \& York, D. G. 2004, Phys. Rev. D, 69, 103501

Tegmark, M., Zaldarriaga, M., \& Hamilton, A. J. 2001, Physical Review D, 63 
Verde, L., Peiris, H. V., Spergel, D. N., Nolta, M. R., Bennett, C. L., Halpern, M., Hinshaw, G., Jarosik, N., Kogut, A., Limon, M., Meyer, S. S., Page, L., Tucker, G. S., Wollack, E., \& Wright, E. L. 2003, ApJS, 148, 195

Wasserman, L. 2004, All of Statistics (New York: Springer-Verlag)

White, M. \& Bunn, E. F. 1995, ApJ, 450, 477 

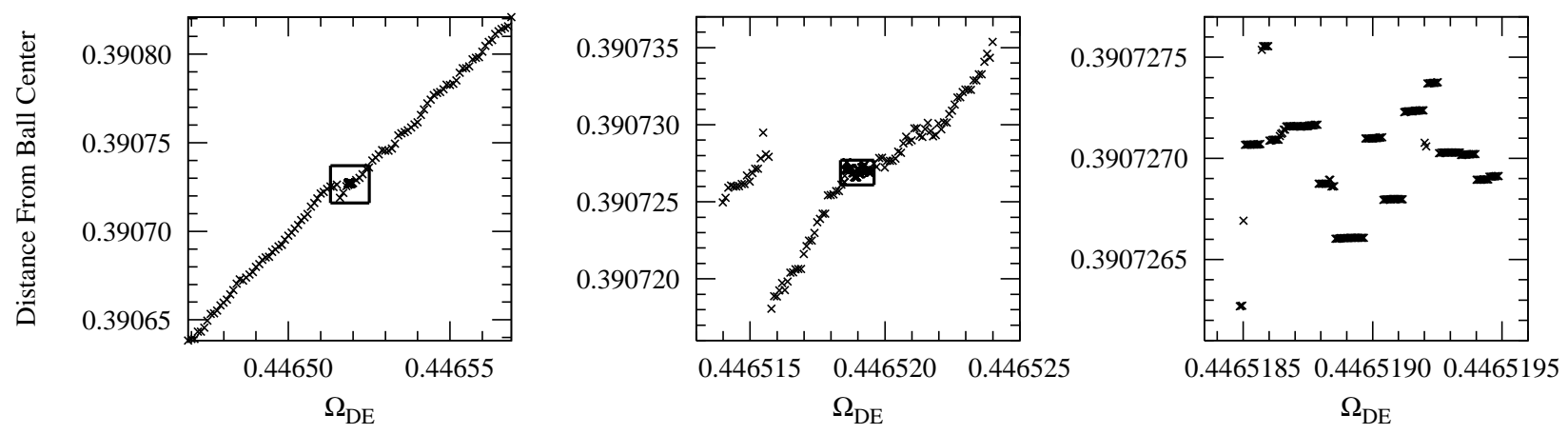

Fig. 10.- A plot of spectra distance as a function of $\Omega_{\mathrm{DE}}$, with all other parameters fixed. The square boxes in each of the left two plots denotes the area enlarged in the neighboring plot to the right. Note that while on the global scales, (A), the mapping appears to be smooth, closer inspection $(\mathrm{B}),(\mathrm{C})$ reveal numerical errors resulting from approximations used in CMBFast. 


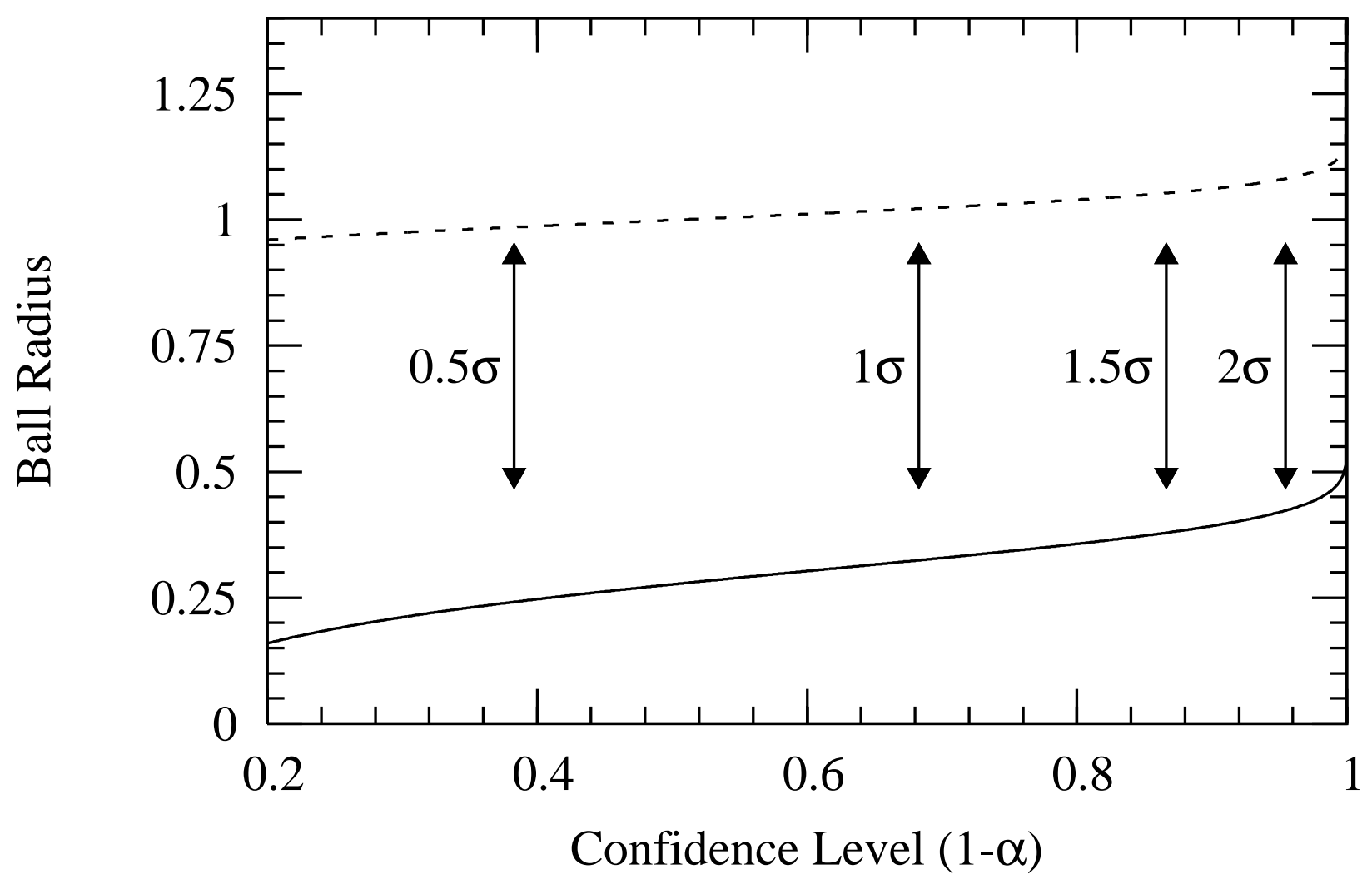

Fig. 11. - Radius of our non-parametric confidence ball as a function of confidence level (solid). The reduced $\chi^{2}$ ball is shown for comparison (dashed). Arrows depict $\frac{1}{2}, 1,1 \frac{1}{2}$ and $2 \sigma$ respectively. 

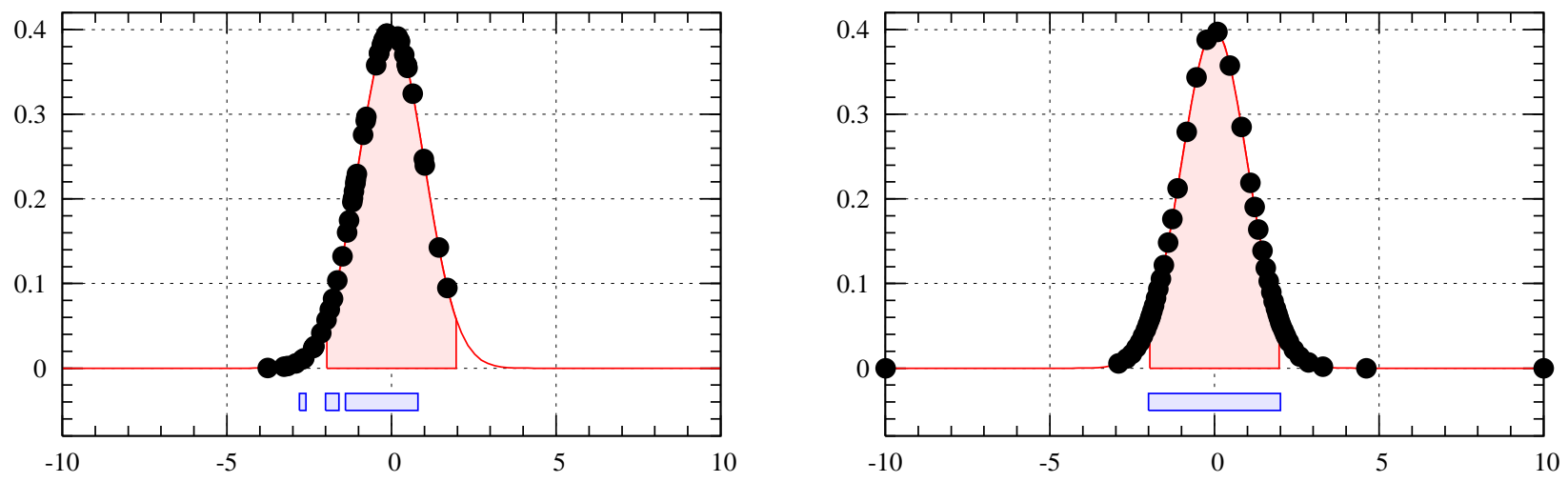

Fig. 12.- Distribution of experiments run by MCMC (left) and our algorithm (right). Black dots denote 50 experiments run in order to determine the $95 \%$ credible / confidence interval (shaded red area) for a standard normal distribution (solid red line). Shaded blue areas below the normal curves indicate the credible / confidence intervals derived for the 50 samples chosen. See the electronic edition of the Journal for a color version of this figure. 